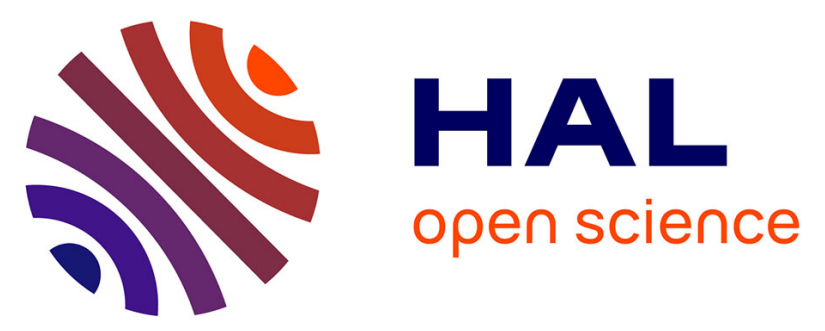

\title{
Toward the Formulation of Stable Micro and Nano Double Emulsions through a Silica Coating on Internal Water Droplets
}

Salman Akram, Xinyue Wang, Thierry F Vandamme, Mayeul Collot, Asad Ur Rehman, Nadia Messaddeq, Yves Mély, Nicolas Anton

\section{To cite this version:}

Salman Akram, Xinyue Wang, Thierry F Vandamme, Mayeul Collot, Asad Ur Rehman, et al.. Toward the Formulation of Stable Micro and Nano Double Emulsions through a Silica Coating on Internal Water Droplets. Langmuir, 2019, 35 (6), pp.2313 - 2325. 10.1021/acs.langmuir.8b03919 . hal03419839

\section{HAL Id: hal-03419839 \\ https://hal.science/hal-03419839}

Submitted on 12 Nov 2021

HAL is a multi-disciplinary open access archive for the deposit and dissemination of scientific research documents, whether they are published or not. The documents may come from teaching and research institutions in France or abroad, or from public or private research centers.
L'archive ouverte pluridisciplinaire HAL, est destinée au dépôt et à la diffusion de documents scientifiques de niveau recherche, publiés ou non, émanant des établissements d'enseignement et de recherche français ou étrangers, des laboratoires publics ou privés. 


\title{
Towards the formulation of stable micro and nano double emulsions through silica coating of internal water droplets
}

\author{
Salman Akram, ${ }^{\dagger}$ Xinyue Wang, ${ }^{\dagger}$ Thierry F. Vandamme, ${ }^{\dagger}$ Mayeul Collot, ${ }^{\#}$ Asad Ur Rehman, ${ }^{\dagger}$ \\ Nadia Messaddeq, ${ }^{\star}$ Yves Mély, ${ }^{\#}$ Nicolas Anton ${ }^{\dagger} * *$ \\ † Université de Strasbourg, CNRS, CAMB UMR 7199, F-67000 Strasbourg, France \\ \$ Université de Strasbourg, IGBMC, Inserm U964, CNRS UMR7104, F-67000 Strasbourg, France \\ \# Université de Strasbourg, CNRS, LBP UMR 7021, F-67000 Strasbourg, France \\ * To whom correspondence should be addressed: Dr. Nicolas Anton, University of Strasbourg, CNRS \\ 7199, Laboratoire de Conception et Application de Molécules Bioactives, équipe de Pharmacie \\ Biogalénique, route du Rhin No.74, F-67401 Illkirch Cedex, France. Tel.: + 333688542 51, Fax: + 33 \\ 3688543 06; E-mail address: nanton@unistra.fr
}

\begin{abstract}
Delivery systems able to co-encapsulate both hydrophilic and hydrophobic species are of great interest for both fundamental research and industrial applications. Water-in-oil-in-water $\left(\mathrm{w}_{1} / \mathrm{O} / \mathrm{W}_{2}\right)$ emulsions are interesting systems for this purpose, but they suffer from limited stability. In this study we propose an innovative approach to stabilize double emulsions by the synthesis of a silica membrane at the water/ oil interface of the primary emulsion (i.e. inner $\mathrm{w}_{1} / \mathrm{O}$ emulsion). This approach allows the formulation of stable double emulsions through a twostep process, enabling high encapsulation efficiencies of model hydrophilic dyes encapsulated in the internal droplets. This approach also decreases the scale of the double droplets up to the nano-scale, which is not possible without silica stabilization. Different formulation and process
\end{abstract}


parameters were explored in order to optimize the methodology. Physicochemical characterization was performed by dynamic light scattering, encapsulation efficiency measurements, release profiles, as well as by optical and transmission electron microscopies.

Keywords: Double emulsions; nano-emulsions; APTES; nanocapsules; drug delivery system. 


\section{Introduction}

Scientific challenges in the fields of agro-food, cosmetic or pharmaceutical sciences have often found innovative solutions in the form of optimized delivery systems. With the unique properties to encapsulate hydrophilic molecules in lipid emulsions, the double emulsions have gained an important place in this research. ${ }^{1}$ In the field of pharmaceuticals, double emulsions can be used as oral delivery systems for vaccines, drugs, and plant bioactives, ${ }^{2,3}$ while in the field of food industry they can be used as carriers for probiotics, vitamins, seasoning agents or extracts. ${ }^{4-8}$ In the field of nanomedicine, nano-scale double emulsions could solvep important challenges, like the targeted delivery of hydrophilic molecules.

When aiming a globule dispersion in aqueous continuous phase, e.g. in most of pharmaceutical formulations, double emulsions are formulated to be in the water-in-oil-in-water type (w/O/W). These systems can be made either through a single-step ${ }^{9,10}$ or a two-step process. ${ }^{11,12}$ In the onestep method, internal and external water phases have the same composition. Moreover, this method may require an additional step of bulk purification, e.g. through a desalination column in the case of nano-sized carriers. This method is not optimized for the encapsulation of high added-value active compounds, and for obtaining high encapsulation efficiencies. On the contrary, in the two-step processes (which we followed), the internal water may be different from the external water, which makes them more suited for optimizing the encapsulation efficiency. ${ }^{13-}$ 15

The primary $\mathrm{w}_{1} / \mathrm{O}$ emulsion must be of very small size, preferably a nano-emulsion stabilized by a low HLB surfactant. Reverse emulsion is generally obtained by a high-energy method, using a high-pressure homogenizer or ultrasounds. For the second emulsification, several approaches can 
be used, but a low-energy method may be privileged in order to prevent the premature release of the hydrophilic material solubilized in the internal water droplets. Indeed, high-energy methods can destroy the primary emulsion, giving rise to simple $\mathrm{O} / \mathrm{W}$ emulsions. ${ }^{16}$

Since many years, double emulsions are considered as an emerging technology with high potential and interest. Therefore, a huge research effort was undertaken to optimize double emulsions for industrial applications and scale-up. However, to date, only a few products based on these emulsions are on the market. ${ }^{4}$ The main issue is their limited stability, i.e. formulating a robust double emulsions able to conserve its specifications over storage time, like appearance, texture and taste, involves long-term physical and chemical stability. This stability is very difficult to control in double emulsions due to the difference in osmotic pressure between the inner and outer water phases that should be equalized, and the difference in Laplace hydrodynamic pressure that tends to vanish with time and modify the morphology of the double structure. These equilibration phenomena are also favored by the surfactants used for the first emulsification, which contribute to destabilize the thin liquid films between internal water droplets. As a result, the stability equation is complex and highly challenging. At this point, using a nano-emulsion as a primary $\mathrm{w}_{1} / \mathrm{O}$ emulsion can be an interesting option, due to the strong stability of the nano-droplets.

On the other hand, stable double emulsions of nanometric size (less than $200 \mathrm{~nm}$ ) are difficult to obtain, because of the second nano-emulsification process very drastic in this case, and the reduced dimension of the oil film between internal droplets and the bulk that can facilitate water transfer. Reducing the scale of the double emulsions should allow nano-encapsulation of hydrophilic and lipophilic species in a single particle, compatible with parenteral and i.v. administration. For example, such a co-encapsulation of drugs is critical for optimizing targeted 
therapies by the simultaneous co-delivery of anticancer species with another complementary cytotoxic molecule, anti-inflammatory agent or adjuvant. Double emulsion formulations at the nanoscale were already reported, ${ }^{12-15,17,18}$ but always by turning one of the liquid phase, i.e. internal water or oil, into a polymerized matrix. Herein, we present for the first time a liquid/liquid/liquid system keeping all advantages of double emulsions in terms of encapsulation and release properties, along with the advantages of nano-emulsions that allow homogeneous dispersion and compatibility with parenteral administration and targeted drug delivery.

In this context, the objective of the present study was to understand the formation of double emulsions obtained with reverse nano-emulsion as a primary $\mathrm{w}_{1} / \mathrm{O}$ emulsion, and to explore how to improve their stability, keeping the liquid/liquid/liquid morphology even at a nanoscale. To improve the double emulsion stability, the internal water/oil interface was reinforced by a silica-based membrane synthesized in situ. ${ }^{19-28}$ It is noteworthy that silica material as well as silica nanomaterials are, in general, widely used in biomedical applications are they are considered as biocompatible and safe. ${ }^{29-31}$ In a first part, micrometric-scale double emulsions were formulated to understand the impact of the formulation parameters on the integrity of the droplets, as monitored by the encapsulation efficiency and leakage of a water-soluble dye (methylene blue). The second part of the study aimed to transpose the optimized formulation to the nanoscale, by modifying the secondary emulsification. Double nano-emulsions were characterized by dynamic light scattering (DLS) and transmission electron microscopy (TEM), as well as by measuring the encapsulation efficiencies (EE) and release profile of hydrophilic model species encapsulated in the internal aqueous droplets. 


\section{Experimental part}

\section{Materials}

Oil compatible with parenteral administration (Labrafac ${ }^{\circledR}$ WL 1349) was obtained from Gattefossé S.A., Saint-Priest, France. This is a mixture of capric and caprylic acids that we used all along this study as oily phase, thus as continuous phase in the primary $\mathrm{w}_{1} / \mathrm{O}$ nano-emulsion. Polyglycerol polyricinoleate (PGPR) was kindly gifted by Stéarinerie Dubois (BoulogneBillancourt, France), and served as lipophilic surfactant (HLB around 1.5) for the preparation of primary $\mathrm{w}_{1} / \mathrm{O}$ nano-emulsion. This emulsifier is largely used for human consumption, being approved for food formulation by the FDA (Food and Drug administration) and the JECFA (Joint FAO/WHO Expert Committee on Food Additives). Kolliphor ELP ${ }^{\circledR}$ (BASF, Ludwigshafen, Germany) is a hydrophilic nonionic surfactant (HLB around 13) compatible with parenteral administration, that was used as hydrophilic surfactant for the second emulsification step in the formulation of double droplets. Methylene blue, tetra ethyl orthosilicate (TEOS), (3Aminopropyl) triethoxysilane (APTES), and sulforhodamine 101 were purchased from Sigma (Saint-Louis, U.S.A.).

\section{Methods}

\section{Formation of primary $w_{1} / O$ nanoemulsion}

The oil phase ( $4.8 \mathrm{~g}$ ) consists of Labrafac ${ }^{\circledR}$ WL 1349 containing PGPR (from 1 to 35 wt.\%) and the silica precursor (quantity of TEOS ranging from 5 to $25 \mathrm{wt} \%$ or APTES from 2.5 to $10 \mathrm{wt} . \%)$. The oil phase was mixed with the aqueous phase $w_{1}(1.2 \mathrm{~g})$ made of distilled water containing $\mathrm{CaCl}_{2}$ (5 wt.\%). This mixture was first vortexed for one minute and then adjusted at 
$50^{\circ} \mathrm{C}$ with a gentle mixing at $100 \mathrm{rpm}$ in a ThermoMixer ${ }^{\circledR} \mathrm{C}$ (Eppendorf) for five minutes. This choice of $50^{\circ} \mathrm{C}$ simply comes from the experiment: compared to the similar experiment at room temperature, stirring the premix at $50^{\circ} \mathrm{C}$ before the stage of the nano-emulsification by ultrasonication, is highly beneficial for the properties of the resulting dispersion. The size distribution is thinner, the sample homogeneity and stability are improved. The reason could be linked to the modification of the phase viscosities lowered at higher temperature, impacting on the phase viscosities ratio, and thus impacting on the critical Weber number $\left(\mathrm{We}_{\mathrm{c}}\right)$ (cf. the classical so-called Grace curve). As a result, viscous forces may prevail over capillary ones and the droplet fractionation is increased. Next, this coarse dispersion was nanoemulsified by ultrasonication (Bio block 75043, Sonics Materials, Newtown, USA) operating at $400 \mathrm{~W}$, $20 \mathrm{kHz}, 3 \mathrm{~mm}$-sonotrode, with total operating times of 5 to 20 minutes (cycles of $30 \mathrm{~s}$ done in succession of $10 \mathrm{~s}$ of sonication and $20 \mathrm{~s}$ off).

\section{Preparation of the micrometric double emulsions $w_{1} / O / W_{2}$}

The $\mathrm{w}_{1} / \mathrm{O} / \mathrm{W}_{2}$ double emulsions of micrometric size were prepared by mixing the aqueous phase $\mathrm{W}_{2}(4.8 \mathrm{~g})$ made of distilled water and Kolliphor ELP $^{\circledR}$ solubilized at concentrations ranging from 15 to $25 \mathrm{wt} . \%$ with the primary emulsion $\mathrm{w}_{1} / \mathrm{O}(1.2 \mathrm{~g})$ playing the role of oil phase in the second emulsification. This mixture was vortexed for $30 \mathrm{~s}$ and emulsified by high speed rotor/stator homogenizer UltraTurrax ${ }^{\circledR}$ (IKA T25M Germany) operating at $15000 \mathrm{rpm}$ for 5 minutes.

\section{Preparation of nanometric double emulsions $w_{1} / O / \mathrm{W}_{2}$}


To formulate double emulsions in the nanometric range, the second emulsification step must be a nano-emulsification process. The oil phase is the primary emulsion $w_{1} / \mathrm{O}(1.2 \mathrm{~g})$ directly mixed with Kolliphor ELP $^{\circledR}$ at different surfactant-to-oil weight ratio (SOR) ranging from 25 to $45 \%$. This mixture was vortexed for $30 \mathrm{~s}$ and finally, the second aqueous phase $\mathrm{W}_{2}(4.8 \mathrm{~g}$ of distilled water) is suddenly added before final vortexing ( 3 minutes). This immediately generates the double nano-emulsion according to the spontaneous emulsification method..$^{32-34}$

\section{Characterization of primary emulsions}

All the characterizations of primary and double emulsions, size, encapsulation efficiency and release, were done in triplicate on different formulations.

Hydrodynamic diameter and PDI. The hydrodynamic diameter, size distribution and PDI of the primary emulsions were determined by dynamic light scattering (Malvern ZS 90, Malvern instrument, Orsay, France). Measurements were performed after a suitable dilution of the sample with oil, up to the point where the milky aspect of the sample becomes transparent, allowing the DLS measurement. In the case of primary emulsions, the diluent was exactly the same oil (medium chain triglycerides, Labrafac ${ }^{\circledR}$ WL 1349 ) used to prepare these primary $\mathrm{w}_{1} / \mathrm{O}$ nanoemulsions. In the second case, $\mathrm{w}_{1} / \mathrm{O} / \mathrm{w}_{2}$, dilution was only performed with distilled water. Effect of dilution on the droplet size distributions was checked by different assays at different dilutions, and (data not shown) in both cases the dilution does not affect the results. Each formulation and measurement were repeated three times. 
Encapsulation efficiency. The encapsulation efficiency of primary emulsions was measured to confirm that the methylene blue dyes are effectively encapsulated in the primary nano-emulsion and can be totally released. The primary emulsion is destabilized by mixing with a large amount of Kolliphor ELP ${ }^{\circledR}$ (with a weight ratio of 50:50 in respect with the primary emulsion) and heated at $90^{\circ} \mathrm{C}$ for one hour (ThermoMixer ${ }^{\circledR} \mathrm{C}$, Eppendorf). Then, the mixture is centrifuged for 30 minutes at 13,500 rpm to complete the destabilization of the $\mathrm{w}_{1} / \mathrm{O}$ nano-emulsion and induce a phase separation. This allows collection of the aqueous phase at the bottom of the tube. The supernatant was removed and the aqueous phase was washed with dichloromethane three times. Finally, dichloromethane was evaporated and the methylene blue was dissolved in distilled water, and quantified by absorption spectroscopy after a controlled dilution (plate reader Safas Xenius $\mathrm{XM}^{\circledR}$ ). The process was repeated three times and averages of encapsulation efficiency were reported as a final value.

\section{Characterization of micrometric double emulsions}

Optical microscopy. The micrometric double emulsions were visually characterized by optical microscopy (AXIO Imager.A1, Carl Zeiss, Marly-le-Roi, France). Different samples and controls were selected: emulsions without primary emulsion, double emulsions without silica precursors, and double emulsions with silica precursors at 2.5 and $10 \mathrm{wt} . \%$ in oil (see details in the text below). A little drop of the suspension was deposited on the glass slide, covered by coverslip, and observed at a magnification of 40x (recorded with an Axiocam camera). This optical characterization reveals the global morphology of the droplets (evidencing the double structure) and their size distribution through a size analysis with ImageJ (done on around 100 droplets). 
Encapsulation efficiency study. The ability of the double emulsions to encapsulate hydrophilic molecules was evaluated through the encapsulation efficiency (EE) value that was determined by comparing the dye encapsulated in the emulsions with the free dye in the bulk aqueous phase. To this end, $1 \mathrm{~mL}$ double emulsion samples were taken in Eppendorf tubes and centrifuged at 13,400 rpm for 30 minutes. As the size of these double droplets is micrometric, their separation is effective by a simple centrifugation. Bulk water was then collected by a syringe, filtered on $0.45 \mu \mathrm{m}$ filters, diluted with distilled water and analyzed by spectrophotometry.

Stability studies at high temperature. To evaluate their stability, the micrometric double emulsions were exposed to $90^{\circ} \mathrm{C}(1 \mathrm{~mL}$ samples in Eppendorf tubes set in a boiler). Stability was assessed by following the EE loss for 30 minutes and 1 hour, as described above.

\section{Characterization of nanometric double emulsions}

Physico-chemical characterization. Hydrodynamic diameter and PDI were determined by dynamic light scattering (NanoZS, Malvern), as described above. In transmission electron microscopy, the double structure of the double emulsion shows a good contrast, mainly because the silica capsules and the oil have two distinct electron attenuations and thus a distinct contrast. Samples were used without any staining agent and were diluted (1/10) with Milli-Q water. A drop of suspension was placed on a carbon grid (carbon type-A, 300 mesh, copper, Ted Pella Inc. Redding, PA) and dried at $60^{\circ} \mathrm{C}$ for 30 minutes. Observations were carried out using a Philips Morgagni $268 \mathrm{D}$ electron microscope operating at $70 \mathrm{kV}$. It is noteworthy that the operating conditions we used $(70 \mathrm{kV})$ are very low compared to TEM experimental conditions generally followed in material science (e.g. $200 \mathrm{kV})$. This was chosen to minimally affect the samples. 
Encapsulation efficiency. As described above for micrometric double emulsions, EE values were determined by quantifying the dye concentration in the external bulk phase. However, as centrifugation is not efficient for separating such nanodroplets, they were separated from free dyes by size exclusion chromatography, using a desalting column (PD-10 Sephadex ${ }^{\circledR}$ G-25 M, GE Healthcare). $1 \mathrm{~mL}$ of sample was introduced into the pre-packed column and then eluted using distilled water (about $30 \mathrm{~mL}$ of distilled water was first introduced into the column for equilibration). The elution showed that double nanodroplets came first, then a clear boundary was observed, finally eluting free dye. The free dye fractions were quantified by spectrophotometry. Each formulation and measurement were repeated three times.

Dye release studies. The release studies were performed by dialysis. $1 \mathrm{~mL}$ of sample was introduced in a dialysis tube (Spectrum labs 12-14 KDa) and dialyzed against distilled water at $37^{\circ} \mathrm{C}$, under gentle stirring at $500 \mathrm{rpm}$, in a volume of $500 \mathrm{~mL}$. The samples were collected after $0.5,1,2,4$ and 6 hours. EE values were determined by separating and quantifying free dyes (by spectrophotometry) and expressed as cumulative release as a function of time.

\section{Spectroscopy}

Emission spectra were recorded on a FluoroMax-4 spectrofluorometer (Horiba Jobin Yvon) equipped with a thermostated cell compartment. For standard recording of fluorescence spectra, the emission was collected $10 \mathrm{~nm}$ after the excitation wavelength. All the spectra were corrected from wavelength-dependent response of the detector. Fluorescent behavior of SR101, in MilliQ water, was recorded as a function of its concentration (data not shown), and showed a linear behavior up to $10-20 \mu \mathrm{M}$, then attenuated at higher concentrations due to a partial quenching of 
SR101, induced by ACQ (aggregation caused quenching) effect. SR101 is included in the inner water at high concentration $\left(100 \mu \mathrm{M}\right.$ in $\left.\mathrm{w}_{1}\right)$ to ensure the dye detectability upon the different formulation stages that involve its dilution. On the other hand, to prove that SR101 is not soluble in oil, it was dispersed in Labrafac ${ }^{\circledR}$ WL 1349 as follows: $2.3 \mathrm{mg}$ of SR101 was mixed in $500 \mu \mathrm{L}$ of Labrafac ${ }^{\circledR} \mathrm{WL} 1349$, heated at $60^{\circ} \mathrm{C}$ for 1 hour, and then slightly centrifugated to remove nondispersed crystals, collecting the supernatant for fluorescent measurement. Fluorescent spectra were done in triplicate on three different formulations, reporting the mean spectra in the figure.

\section{Results and discussion}

The great interest of double emulsions, even today, remains undeniable, probably due to the lack of appropriate solutions to encapsulate hydrophilic materials, or to co-encapsulate hydrophilic and lipophilic materials. However, since the double emulsions are of limited stability, the present study proposes a simple modification of the conventional two-step preparation process to stabilize them by reinforcing the $\mathrm{w}_{1} / \mathrm{O}$ interface with a small silica membrane. This innovative methodology not only makes it possible to create stable micrometric double emulsions (of about $10 \mu \mathrm{m})$, but also to formulate double nanodroplets under $130 \mathrm{~nm}$ in diameter. The formulation process as well as the structure of the formulated double droplets are described in Fig. 1. 


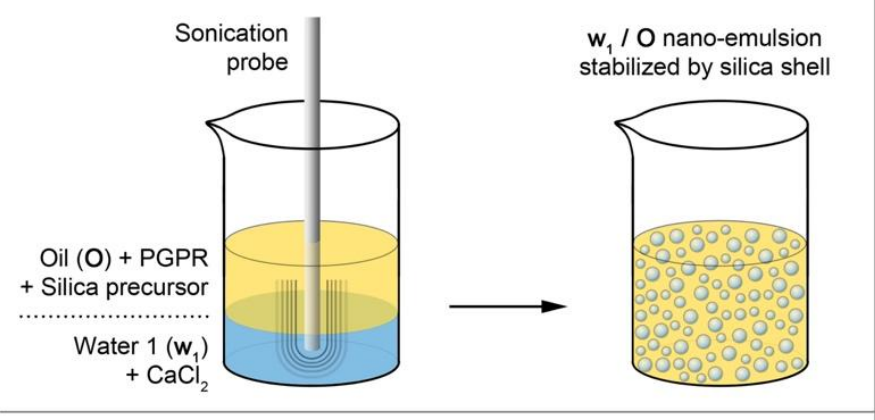

Second emulsification: Rotor / stator or Spontaneous emulsification

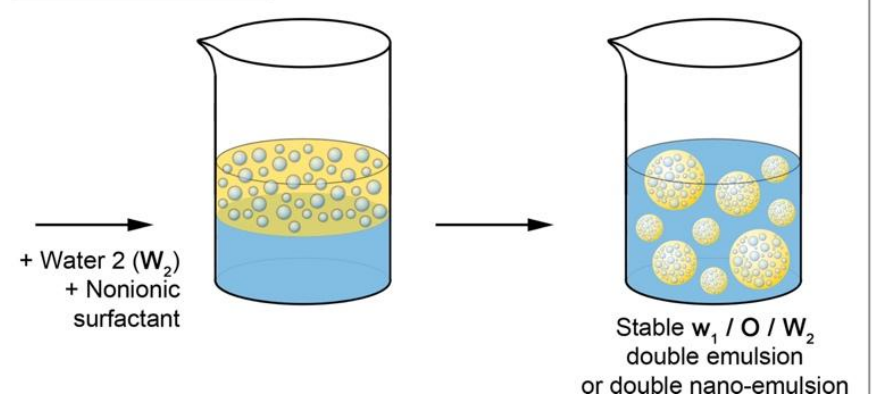

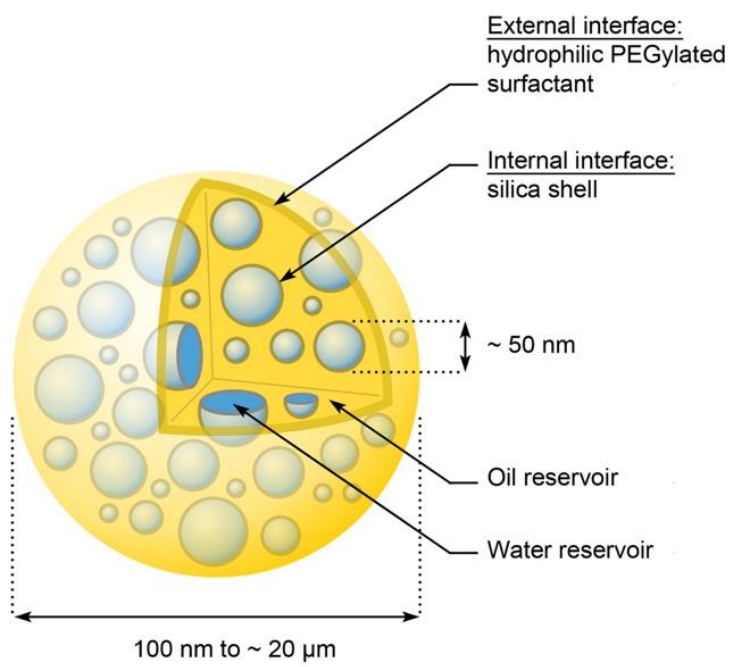

$100 \mathrm{~nm}$ to $\sim 20 \mu \mathrm{m}$

Figure 1: Formulation of the micrometric and nanometric double droplets stabilized by a silica shell. The first emulsification provides the primary reverse nano-emulsion for which the water / oil interface is stabilized by both PGPR and silica shell. The Second emulsification is performed either by a rotor / stator apparatus (Ultraturrax ${ }^{\circledR}$ ) for micrometric double emulsions, or by spontaneous emulsification for double nano-emulsion.

For the formulation of the first emulsion, the oil phase contains a lipophilic surfactant and a silica precursor. The energy supplied by the sonication process has two roles $(i)$ it allows the creation of the nano-droplets, and (ii) it induces the polycondensation of the silica precursor $^{19-}$ ${ }^{22,35}$ specifically at the water / oil interface. As a result, this first process produces aqueous nanodroplets in the form of silica nano-capsules. ${ }^{36,37}$ Next, this primary emulsion is used as oil phase for a second emulsification process, in order to generate a micrometric double droplets by using a mechanical method (rotor / stator, Ultraturrax ${ }^{\circledR}$ ), or nanometric double droplets by spontaneous emulsification. ${ }^{32,38}$ In the following sections, we will investigate in depth the formulation of the 
primary emulsion, and analyze the impact of the formulation parameters on its properties, and then we will characterize the macro-scale and nano-scale double emulsions.

\section{Primary $w_{1} / O$ nano-emulsions and silica nanocapsules}

The singularity of the nano-emulsification process based on ultra-sonication lies in the amount of energy focused on a small volume. The high acoustic field leads to the gradual growing of cavitation bubbles, up to their implosion. This induces huge shearing forces that break-up the aqueous droplets into smaller ones that are stabilized by the surfactants (PGPR). The average droplet size decreases as a function of the quantity of energy supplied.

To prevent the destruction of the double droplets during and after the second emulsification, the original approach proposed in this study resides in the stabilization of the water / oil interface of the primary droplets by a silica membrane synthetized in situ, to preserve the aqueous droplets and their encapsulation properties. Based on previous work ${ }^{19-22}$ the silica precursors were introduced in the oily phase, and the interfacial poly-condensation was initiated through a sonochemistry-mediated reaction. Two different silica precursors, TEOS and APTES were studied. The growth of the silica chains occurs by simultaneous presence of water and precursors solubilized in oil, the silica shell is therefore formed and trapped at the water / oil interface.

\section{Reverse nano-emulsification}

In this section, we will focus on the emulsification itself without silica and investigate the influence of the surfactant concentration, and processing time, on the size and polydispersity of the primary $\mathrm{w}_{1} / \mathrm{O}$ nano-emulsions. By monitoring the impact of the sonication time for different

PGPR concentration $\left(\phi_{V}^{\text {water }}=20 \%\right)$, we observed as expected, ${ }^{39-42}$ a decrease of the droplet 
size (fig. 2a) according to a monoexponential decay $d=A e^{(-t / \tau)}+d_{\infty}$ (where $d$ is the droplet diameter, $A$ is a constant, $d_{\infty}$ the saturation diameter (diameter after stabilization of the monoexponential function), $t$ the sonication time, and $\tau$ the characteristic time of the exponential). The choice of this monoexponential model was based on literature, considered as the more accurate description of the droplet size decrease, as a consequence of the droplet fractionation induced by ultrasonication. ${ }^{39}$ The droplet fractionation directly results from the amount of energy supplied, and gradually stabilizes up to a saturation size $d_{\infty}$. The droplet size is clearly dependent of the surfactant concentration, with a decrease by about $800 \mathrm{~nm}$ between $1 \mathrm{wt} . \%$ and $35 \mathrm{wt} . \%$. Reporting the values of the characteristic time $\tau$ as a function of the sonication time (Fig. 2 (b)) revealed that the time necessary to stabilize the droplet size showed first (up to $7.5 \mathrm{wt} \%$ ) a plateau giving $\tau \sim 30 \mathrm{~min}$, indicating that the nano-droplets are rapidly stabilized independently to the PGPR concentration. ${ }^{38,43,44}$ Then, a second regime appears (from $10 \mathrm{wt} . \%$ ) where the $\tau$ values are much higher and gradually increase up to $100 \mathrm{~min}$. This regime likely corresponds to a saturation regime, where the droplets are rapidly stabilized after their formation, but followed by a further evolution of the size much slower. The threshold of $10 \mathrm{wt} \%$ PGPR is believed to correspond to the saturation regime that allows to reach very small sizes. When extrapolating the curve fits at $t=0$ (Fig. 2 (c)), the sizes also show a stabilization for PGPR concentrations higher than 10-15 wt.\%. The $y$-intercept reported in Fig. 2 (c) is an interesting parameter reflecting the whole behavior of the emulsification process, thus can allow a comparison between the different PGPR concentrations. 
(a)

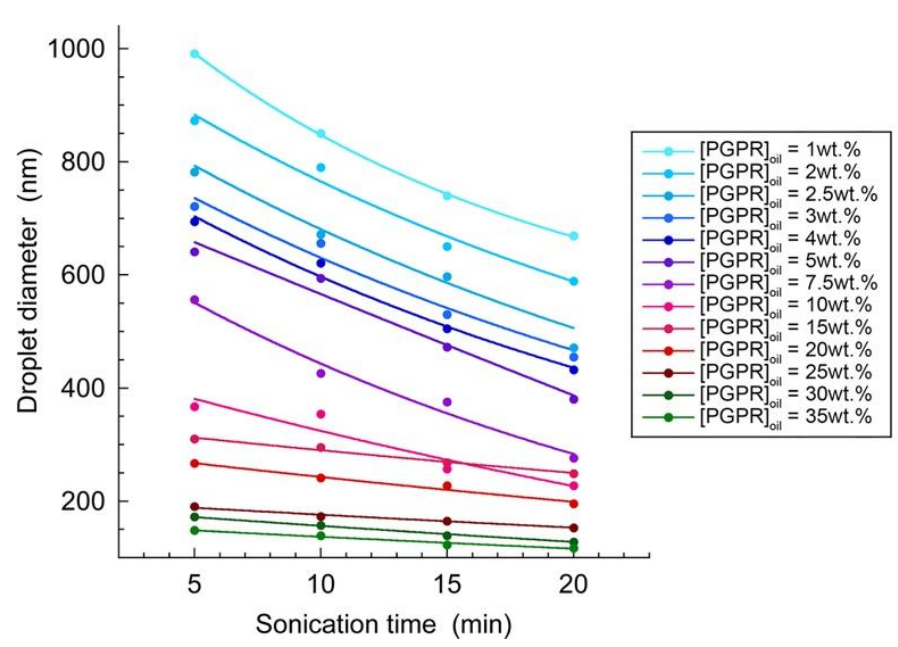

(b)

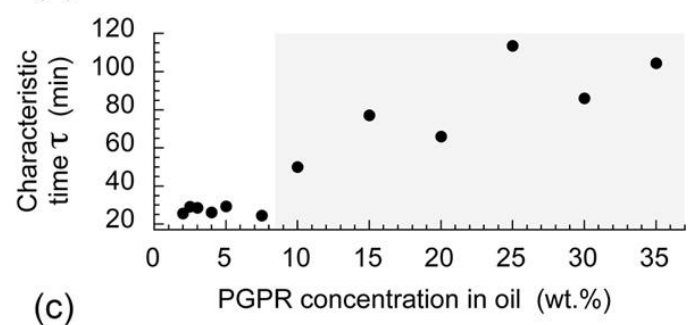

(c)

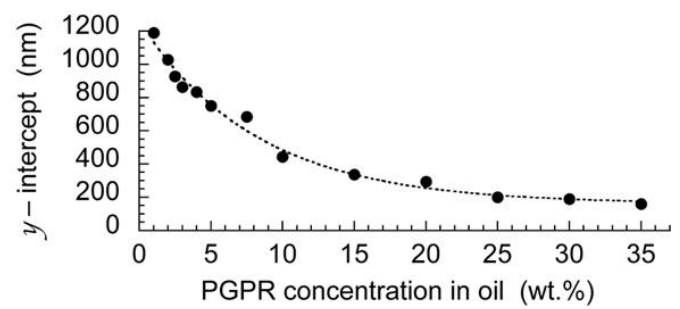

Figure 2: Dependence of the size of $\mathrm{w}_{1} / \mathrm{O}$ nano-emulsion as a function of time and concentration of surfactant (PGPR) in oil. (a) The curves of the dependence of the size of $\mathrm{w}_{1} / \mathrm{O}$ nano-emulsion ( $\phi_{V}^{\text {water }}=20 \%$ ), as a function of the sonication time were fitted by an exponential model (see details in the text). (b) Characteristic time and (c) $y$ intercept from the curve fits of (a) $v s$. surfactant concentration.

In order to formulate reverse nano-emulsions below $300 \mathrm{~nm}$, our data pointed out that a PGPR concentration in oil of at least $10 \mathrm{wt} . \%$ is required. However, a sonication time of 20 minutes is too long for a potential industrial application, as it may destroy the encapsulated materials, increase the sample temperature, and release a high amount of titanium nanoparticles from the sonication nip. An ideal sonication time should be no more than 5 minutes. In addition, to obtain double nano-emulsions based on this primary emulsion, the droplet size should be ideally decreased to $100 \mathrm{~nm}$ or less. ${ }^{45,46}$

Accordingly, the sonication time was fixed to $5 \mathrm{~min}$, and the PGPR concentration was varied from 10 wt.\% to 35 wt.\%. The results reported in Fig. 3 confirmed the trend revealed in Fig. 2, with a significant size decrease as a function of the surfactant concentration. At $[\mathrm{PGPR}]_{\text {oil }}>$ 25 wt.\%, sizes below $160 \mathrm{~nm}$ were observed. However, the variability of the results was quite 
high, likely related to the polydispersity of the nano-emulsions, since PDI values ranged from 0.3 to 0.5 for all measurements with pure water reported in Fig 3. In line with previous reports ${ }^{44,47}$ showing that the emulsifying properties of PGPR can be improved when calcium chloride is added in the aqueous phase, the emulsification process was clearly more efficient in the presence of this additive (Fig. 3, open symbols), providing droplets below $100 \mathrm{~nm}$ for [PGPR] $\geq 10 \mathrm{wt} . \%$, and down to $50 \mathrm{~nm}$ for $[\mathrm{PGPR}] \geq 30 \mathrm{wt} . \%$. Moreover, calcium chloride also improves the homogeneity and reproducibility (standard deviations are between 3 and $10 \mathrm{~nm}$ ) of the results, as well as a strong improvement of the monodispersity (PDI values is equal to $0.16 \pm 0.10$ for $[\mathrm{PGPR}]=10 \mathrm{wt} . \%$, and to $(0.06 \pm 0.05)$ for $[\mathrm{PGPR}] \geq 25 \mathrm{wt} . \%)$.

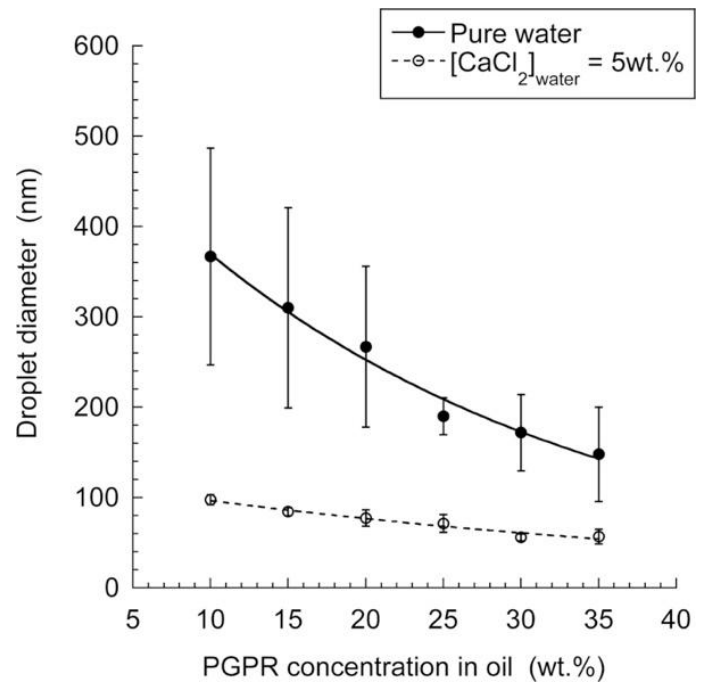

Figure 3: Effect of the PGPR concentration on the size of $\mathrm{w}_{1} / \mathrm{O}$ nano-emulsions at a fixed sonication time of $5 \mathrm{~min}$ and $\phi_{V}^{\text {water }}=20 \%$. The experiments were done with pure distilled water as aqueous phase (Pure water) and using water-containing calcium chloride as additive $\left(\left[\mathrm{CaCl}_{2}\right]_{\text {water }}=5 \mathrm{wt} . \%\right)(\mathrm{n}=3)$. Lines are drawn to guide the eye.

The strong effect of $\mathrm{CaCl}_{2}$ on the emulsification process may be a consequence of the modification of PGPR activity. Indeed, $\mathrm{CaCl}_{2}$ is a chaotropic salt ${ }^{48}$ that affects the hydrogen 
bounding between the water and the PGPR molecules. As a result, the lipophilicity of the emulsifier may be increased and its HLB lowered. In addition, $\mathrm{CaCl}_{2}$ can also directly affect the interfacial tension and improve the emulsification properties. ${ }^{44,47,49}$ In these articles, the authors have shown that the addition of $\mathrm{CaCl}_{2}$ in water can decrease the droplet size and improve the stability of the primary $\mathrm{w}_{1} / \mathrm{O}$ emulsion. This was explained by a specific action on the hydrophilic part of amphiphile like PGPR, through an increase of surfactants adsorption density, giving rise to decreasing interfacial tension along increasing the interfacial elasticity, and as well involving a reduction in the attractive forces between water droplets after their formation. At final, all these effects combined can strongly impact on the emulsification process, lowering the rate of droplet recombination during the sonication, and thus decreasing size, polydispersity, and variability of the results, i.e. lowering standard deviation as it is presented in Fig. 3. To conclude, in view of these results the formulation with $[\mathrm{PGPR}]_{\mathrm{oil}}=30 \mathrm{wt} . \%$ (giving a droplet size of $(57 \pm 4) \mathrm{nm}$ and a PDI value equal to 0.06$)$ was selected as the primary emulsion for the fabrication of the double emulsions.

\section{Silica nano-capsules}

As described above, the silica shell is built at the water/oil interface of the reverse nanoemulsions through sonochemistry. ${ }^{19-22,35}$ Two different silica precursors (either TEOS or APTES) solubilized in the oil phase. The silica precursors hydrolyze first and then undergo the condensation process. The hydrolysis of the APTES and TEOS leads to the production of ethanol and trisilanols. The $\mathrm{Si}-\mathrm{C}$ bond will not be hydrolyzed because this bond is hydrolytically stable, and thus aminopropyl group will stay in the polymer structure. On the other hand, the transient silanol groups will condense with other silanols to produce the amino functionalized network. 
Interestingly, the impact on the double emulsion of the alcohol formed during the chemical reaction, cannot be specifically determined, but will be included in the global determination of the encapsulation efficiency values, along with the other formulation and process parameters. The emulsification process was performed in the same conditions as described above: $\phi_{V}^{\text {water }}=$ $20 \%$, sonication time $=5 \mathrm{~min}$, and $[\mathrm{PGPR}]_{\mathrm{oil}}=30 \mathrm{wt} . \%$. The sizes of the reverse nano-droplets (Fig. 4) were compared for the APTES and TEOS precursors.

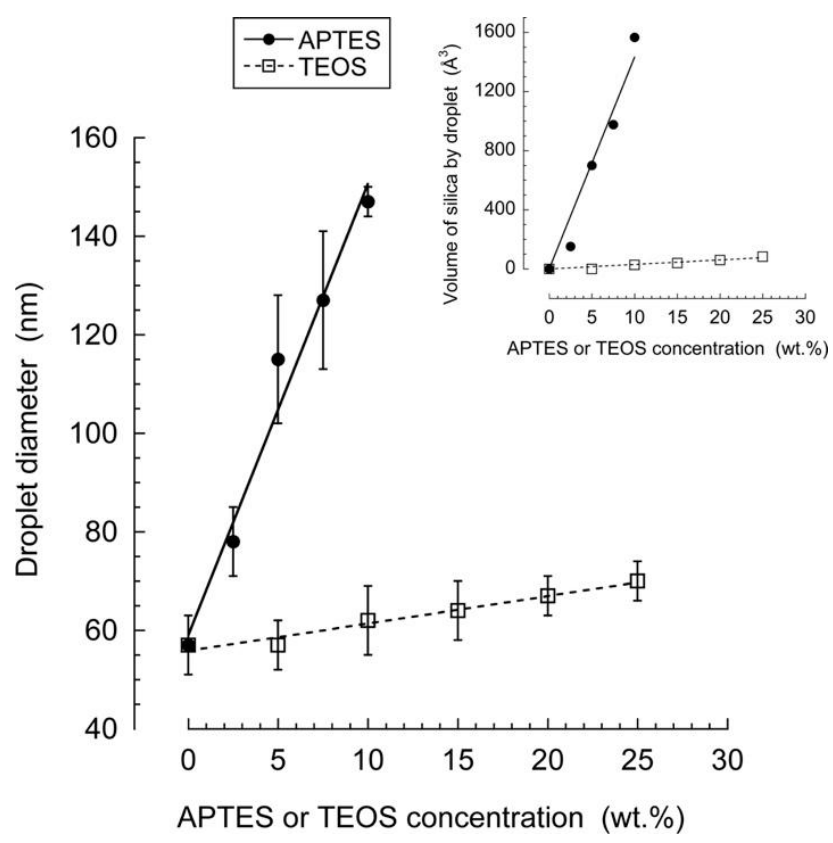

Figure 4: Effect of the concentration of silica precursor (either APTES or TEOS) on the size of the primary $w_{1} / \mathrm{O}$ nano-emulsion $(\mathrm{n}=3)$. Inset: Dependence as a function of the silica precursor of the volume of the nanodroplets, calculated from their diameter. 
In both cases, the introduction of the silica precursor in oil before the sonication increases the size of the water droplets, likely due to the formation of a silica shell around the droplets. Moreover, in both cases, the shell thickness is linearly related to the concentration of silica precursor in oil. Nevertheless, the slopes are different because, probably, a part of silica still remains in oil or has been expelled in water. It is important to note that we cannot prove that the water droplets do not increase when the silica precursor amount is increased, however, we have thus assumed that, since the formulation parameters were fixed $\left(\phi_{V}^{\text {water }}=20 \%\right.$, sonication time $\left.=5 \mathrm{~min},[\mathrm{PGPR}]_{\mathrm{oil}}=30 \mathrm{wt} . \%\right)$, the size of the water droplet core is considered constant and the silica shell gradually growing around. In addition, the fact that the relationship between droplet size and silica precursor concentration is linear, supports the assumption that the size of the water cavities should have the size of the initial aqueous droplets without silica (around $60 \mathrm{~nm}$ in the example shown in Fig. 4), and the silica shells growth around it.

However, APTES appears much more efficient than TEOS for the interfacial polycondensation. For example, at a concentration of $10 \mathrm{wt} \%$, TEOS induced a shell thickness of about $3 \mathrm{~nm}$, while it reached about $45 \mathrm{~nm}$ with APTES. Based on these data, the global volume of the silica shell can be estimated as a function of the precursor concentration (Fig. 4 Inset). The comparison of the growth rates (slopes of the linear fits, 3.0 and $143.2 \AA^{3}$.wt.\% $\%^{-1}$ for TEOS and APTES, respectively) indicates that 50 times more material is synthesized at the interface with APTES as compared to TEOS. The silica shell appears thus important to stabilize the primary emulsion in order to formulate the double droplets, and these results demonstrated here the feasibility of the in situ synthesis of the silica shell in the water/oil interface of the droplets. The linear relationship between the shell thickness and the concentration of the silica precursor indicates that the shell thickness can be adjusted very finely, and thus, control the encapsulation and 
release properties of the double emulsions. The reason why APTES is more efficient than TEOS in the polycondensation process may be related to its chemical structure. ${ }^{50}$ Unlike TEOS, the amphiphilic properties of APTES likely play an active role in their concentration at the water / oil interface. Indeed, since the pKa of APTES, $\left(\mathrm{CH}_{3}-\mathrm{CH}_{2}-\mathrm{O}\right)_{3}-\mathrm{Si}-\left(\mathrm{CH}_{2}\right)_{3}-\mathrm{NH}_{2}$, is around 8.5, and the aqueous phase containing $\mathrm{CaCl}_{2}$ has a $\mathrm{pH}$ slightly below 7 , the protonation of the amino function probably accelerates the migration of APTES (from oil) towards the interface, increasing the efficiency of the ultrasound-mediated interfacial polycondensation. In contrast, TEOS, lacking such a function, $\left(\mathrm{CH}_{3}-\mathrm{CH}_{2}-\mathrm{O}\right)_{4}-\mathrm{Si}$, may encounter the interface only because of molecular diffusion.

\section{Micrometric double emulsions $\mathrm{w}_{1} / \mathrm{O} / \mathrm{W}_{2}$}

To prove that double droplets are stable when the inner droplet interface is reinforced by a silica membrane, the micrometric double emulsions formulated by a rotor / stator (Ultraturrax ${ }^{\circledR}$ ) device were characterized for their aspect, morphology and size by optical microscopy (Fig. 5). The microscopy images show the controls without primary emulsions $\mathrm{O} / \mathrm{W}_{2}$ (Fig 5a), the double emulsions $\mathrm{w}_{1} / \mathrm{O} / \mathrm{W}_{2}$ without silica precursor (Fig $5 \mathrm{~b}$ ), the double emulsions $\mathrm{w}_{1} / \mathrm{O} / \mathrm{W}_{2}$ with 2.5 wt.\% APTES (Fig 5c), and the double emulsions $w_{1} / \mathrm{O} / \mathrm{W}_{2}$ with $10 \mathrm{wt} \%$ APTES (Fig 5d). 

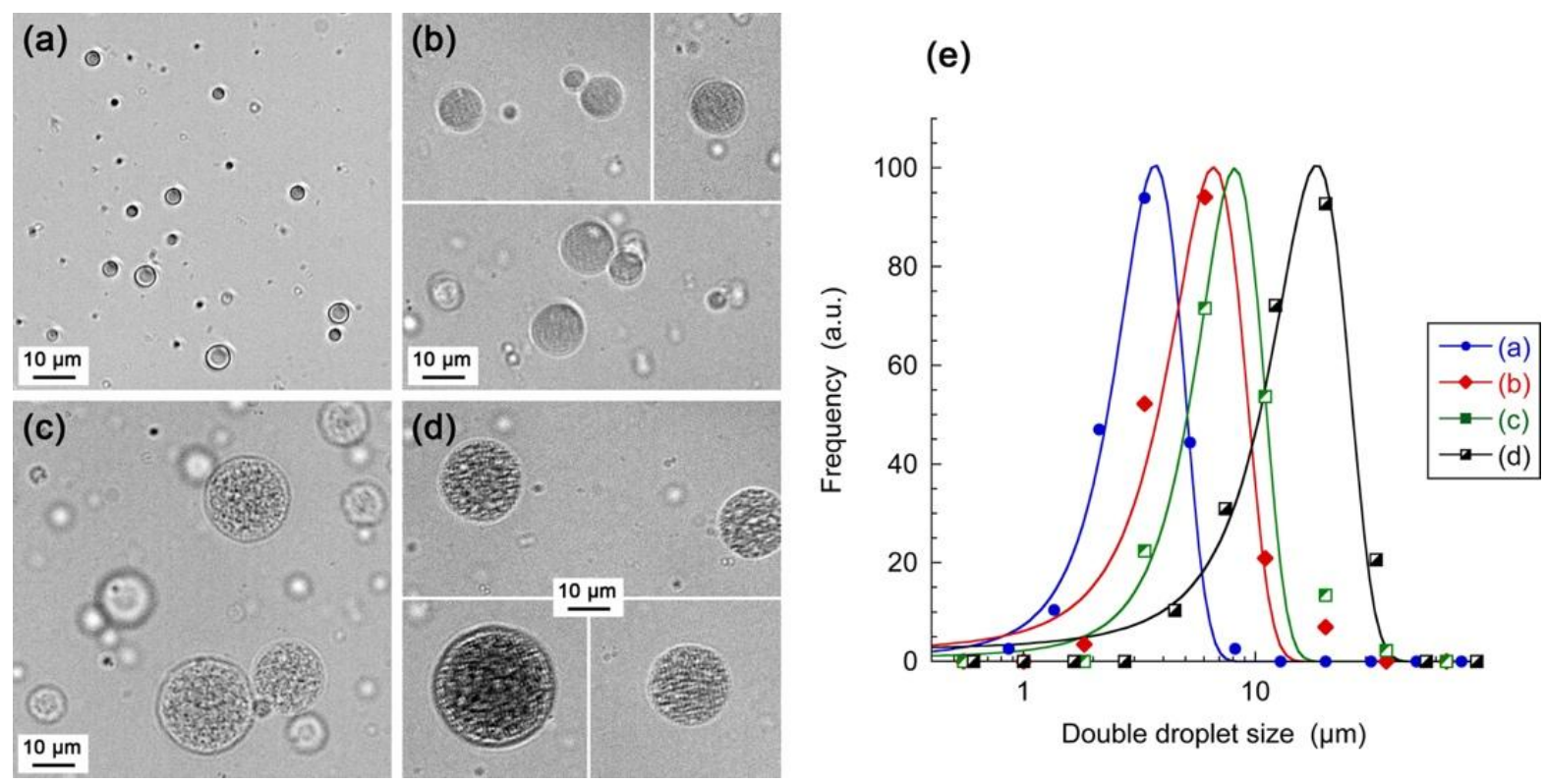

Figure 5: Optical microscopy of controls without primary emulsions $\mathrm{O} / \mathrm{W}_{2}(\mathrm{a})$, the double emulsions $\mathrm{w}_{1} / \mathrm{O} / \mathrm{W}_{2}$ without silica precursor (b), the double emulsions $\mathrm{w}_{1} / \mathrm{O} / \mathrm{W}_{2}$ with 2.5 wt.\% APTES (c) and with 10 wt.\% APTES (d) $\left(\phi_{V}^{\text {oil phase }}=20 \%\right.$ and $\left[\right.$ Kolliphor $\left.\left.\mathrm{ELP}^{\circledR}\right]=25 \%\right)$. (e) Corresponding size distribution analysis on samples of hundred droplets and fitted by a Gaussian function.

The double droplets as well as the control exhibit a quite homogeneous distribution. While in the control (a), the oil droplet core appears clear and transparent, the primary emulsion is clearly visible as small "corrugations" within the double droplets in (b), (c) and (d). These corrugations are likely due to the light diffraction of the aqueous internal droplets and the limited resolution of the optical microscope around $300 \mathrm{~nm}$. Based on these images, the double droplet size distribution was determined by a graphical analysis (Fig. 5, right) for about hundred droplets each time. The experimental data were successfully fitted with Gaussian equation, giving average sizes of $3.7 \mu \mathrm{m}, 6.6 \mu \mathrm{m}, 8.1 \mu \mathrm{m}$ and $18.3 \mu \mathrm{m}$, respectively for (a), (b), (c) and (d). As expected, the emulsification process is affected by the presence and size of the primary droplets. 
Indeed, since the size of these droplets playing the role of template increases with increasing APTES concentration, the size of the double droplets, increases as well.

To investigate the stability of these double emulsions upon the formulation process, we monitored the leakage of methylene blue (MB) solubilized in the inner water $\mathrm{w}_{1}$, towards the external water $\mathrm{W}_{2}$. It is important to note that the $\mathrm{EE}$ values are those immediately after the second emulsification, and thus describe the impact of the process itself on the structure of the double droplets, while the leakage of $\mathrm{w}_{1}$ induced by storage will be studied and discussed in the following sections. Before discussing the $\mathrm{EE}$ values of double droplets, it is important to consider the preliminary controls regarding the full release of MB from the primary emulsion. In these control experiments performed on the primary emulsions (described in the experimental section), for APTES varying from 0 to $10 \mathrm{wt} \%$ and for TEOS varying from 0 to $25 \mathrm{wt} . \%$, values of total release of $(99.6 \pm 0.2) \%$ and $(99.2 \pm 0.4) \%$, respectively. This clearly demonstrates that encapsulated materials can be released irrespective of the thickness of the silica shell. 

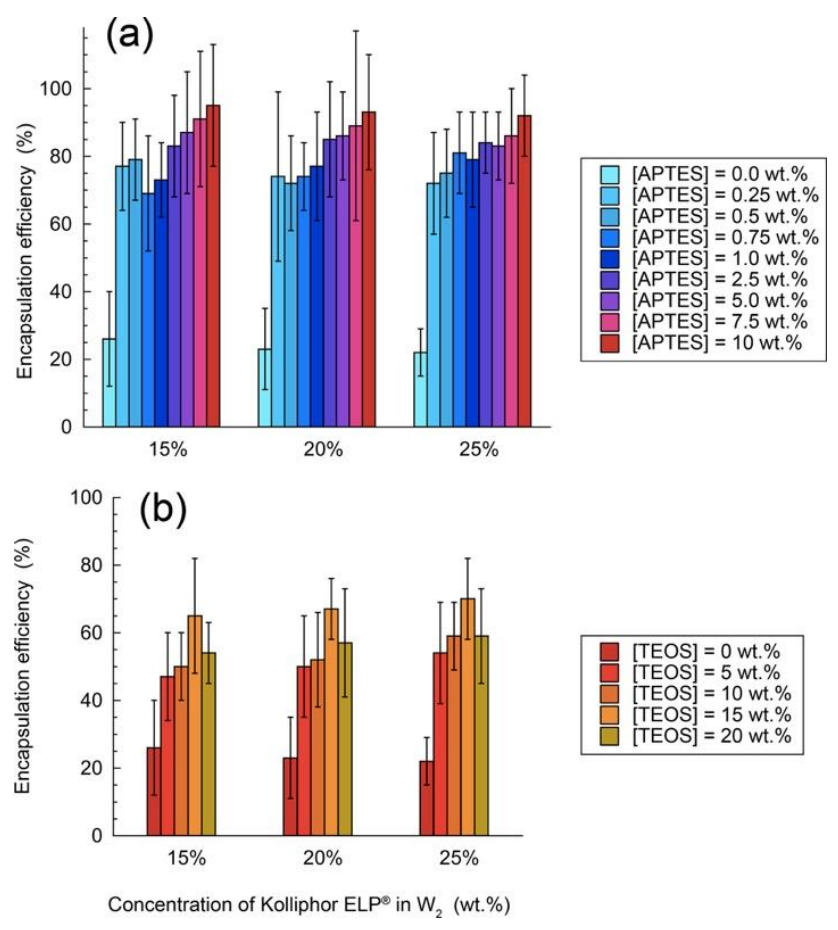

Figure 6: Encapsulation efficiency of MB solubilized in the inner water droplets of macroscopic double emulsions, immediately after the second emulsification. Effect of the concentration of the silica precursor in oil (APTES in (a), and TEOS in (b)), and for different concentrations of the hydrophilic surfactant (Kolliphor ${ }^{\circledR}$ ELP) in $\mathrm{W}_{2}(\mathrm{n}=3)$.

The EE values for the double emulsions formulated with APTES and TEOS are reported in Fig. 6, for different concentrations of the hydrophilic surfactant (Kolliphor ${ }^{\circledR}$ ELP). The EE value for the formulation without silica is relatively weak ( 25\%), but increases rapidly with the silica concentration. In the case of APTES, the EE value increases rapidly to $80 \%$ for $[\mathrm{APTES}]=0.25 \mathrm{wt} . \%$ and reaches about $95 \%$ for $[\mathrm{APTES}]=10 \mathrm{wt} . \%$. A similar trend is observed for TEOS, but higher TEOS concentration are needed and EE values hardly exceed 60\%. These results are in line with the respective efficiencies of APTES and TEOS in the fabrication of the silica shell (Fig. 4). They indicate that even at very low concentrations, APTES can reinforce efficiently the water/oil interface to stabilize the double emulsion against the 
second emulsification. The strong effect of low concentrations of APTES on the EE is outstanding and discloses the high potential of the technology to produce stable double emulsions compatible with the regulatory aspects imposed by industrial production and applications. The difference in EE between APTES and TEOS is likely related to the thickness and probably the density of the silica shell (Fig. 4), which makes the silica shell more impermeable with APTES. In fact, in Fig. 4 inset, the "volume of silica by droplet" is represented in function of the precursor concentration, revealing an important difference between APTES and TEOS, thus a parallel can be drawn with the EE values correlating the results of Fig. 6. Due to its high efficiency, we selected APTES for further stability studies and the formulation of double droplets at the nano-scale. It is noteworthy that the "stability" herein claimed and brought by the reinforcement of interface by silica, refers to the fact that the internal water droplets are much more robust compared to the ones without silica. The globules formed are much more robust towards changes in temperature, changes in osmotic pressure, and also towards the formulation processes (high differences in EE when we compare with and without silica). As a result, we can say that classical double emulsions are much more fragile compared to these new "stabilized" formulations. This is all the more confirmed by the accelerated ageing experiment detailed below.

To further investigate the stability of the double droplets, we monitored their EE values at high temperature $\left(90^{\circ} \mathrm{C}\right)$ as a function of time and for several APTES concentrations (Fig. 7). The idea here was to place the samples in very drastic conditions, like accelerated ageing procedures are generally worked out (to a lesser extent). The results appear in line with our expectation, exhibiting a significant difference between control and silica-protected samples. The temperature of $90^{\circ} \mathrm{C}$ was in fact chosen as the higher temperature before boiling to conduct such experiments 
in drastic temperature conditions. For the control (without APTES), the EE value starts at $23 \%$, and falls down to $0 \%$ after $30 \mathrm{~min}$, indicating a very rapid release of the encapsulated compounds. In contrast, for $[$ APTES] $\geq 0.25 \mathrm{wt} . \%$, the double droplets appear much more resistant. A slow and regular release of dyes was observed, whatever the amount of silica. This observation confirmed the strengthening of the interface by the silica membrane, even at 0.25 wt. $\%$ APTES, and the still possible release of encapsulated materials with time, as evidenced by the $20-30 \%$ decrease in the EE value after $60 \mathrm{~min}$. After one hour, the encapsulated MB became purple (the color of the crystalline form of this molecule) probably as a result of crystallization, which did not allow this experiment to be continued at $90^{\circ} \mathrm{C}$.

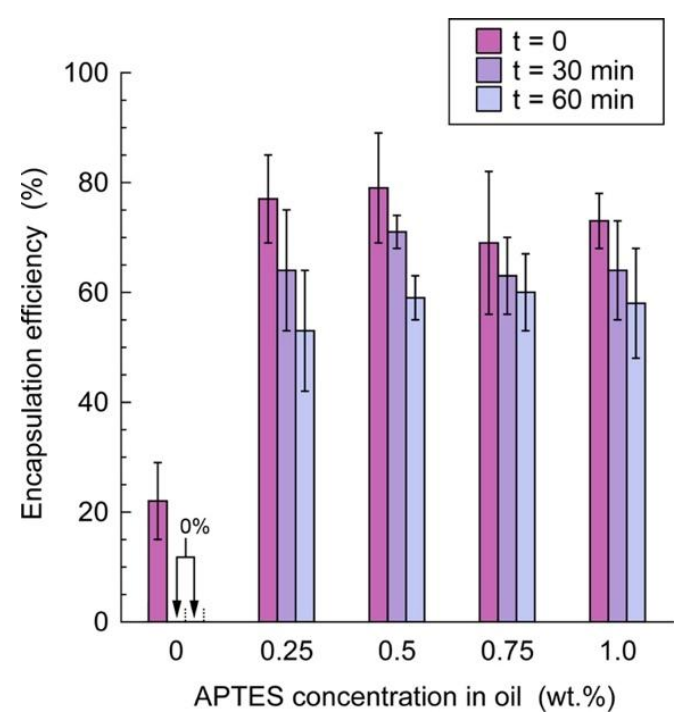

Figure 7: Encapsulation efficiency of MB solubilized in the inner water droplets of macroscopic double emulsions $\left(\phi_{V}^{\text {oil phase }}=20 \%\right.$ and $\left[\right.$ Kolliphor $\left.\left.\operatorname{ELP}^{\circledR}\right]=25 \%\right)$, immediately after the second emulsification $(\mathrm{t}=0)$ or kept at high temperature $\left(90^{\circ} \mathrm{C}\right)$ for 30 or 60 minutes. The $[\text { APTES }]_{o i l}=0 \mathrm{wt} . \%$ data point corresponds to the control without silica precursor. 
These results indicate that the silica shell formed by APTES is resistant to high temperature, which opens a number of applications. For example, in food applications, the double emulsions can ensure an efficient storage of hydrophilic materials like flavors, and then allow their controlled delivery during cooking.

\section{Nanometric double emulsions $\mathrm{w}_{1} / \mathrm{O} / \mathrm{W}_{2}$}

In this last section, our goal was to transfer the best experimental conditions described for double micron emulsions to the formulation of double droplets smaller than one micrometer. For this purpose, the second emulsification has been adapted to be a nano-emulsification process capable of preserving the structure of the primary $\mathrm{w}_{1} / \mathrm{O}$ nano-emulsions. Basically, nano-emulsification methods are divided into high energy processes $^{51}$ (high-pressure homogenization or utrasonification), and low-energy processes based on spontaneous emulsification. To preserve the primary emulsion, we selected the gentlest process, i.e. the spontaneous emulsification. In this process, the hydrophilic surfactant in the oily phase $\left(\mathrm{w}_{1} / \mathrm{O}\right.$ nano-emulsion) is mixed at room temperature, and when homogenized, the second aqueous phase $\mathrm{W}_{2}$ is added, giving spontaneous nano-emulsification. ${ }^{52,53}$ Though it is a soft process, the droplets are formed as a result of the turbulent penetration of water in the network made of oil, primary droplets and Kolliphor ELP ${ }^{\circledR}$. This turbulent droplet formation is less destructive than high-energy methods but is nevertheless aggressive for the small aqueous nano-droplets of the primary emulsion. This is confirmed by the data in Fig. 8 correlating the size of the nanometric double emulsion (Fig. 8 (a)) with the encapsulation efficiency of MB (Fig. 8 (b)), as a function of the surfactantto-oil weight ratio (SOR) and the APTES concentration (i.e. thickness of the silica shell). The global size of the double nano-emulsions is below $120 \mathrm{~nm}$, and as expected, decreases as the 
quantity of surfactant increases, reaching $70 \mathrm{~nm}$ for $\mathrm{SOR}=40 \%$. The general behavior of the size decrease observed is similar whatever the concentration of APTES, indicating that the spontaneous emulsification is robust enough to not be affected by the modification of oil composition. The EE values (Fig. 8 (b)) are strongly impacted by the silica shell. Indeed, without silica ([APTES] $=0 \mathrm{wt} . \%$ ), all the MB leaks rapidly and the EE value is close to zero. Then, the EE values regularly grow as the APTES concentration in oil increases, reaching 60-70\% for $[\mathrm{APTES}]=10 \mathrm{wt} . \%$. Compared to macro-scale double emulsions, the nano-emulsification process has a real destructive effect on the double nano-droplets, and thus requires a higher shell thickness to provide efficient encapsulation (i.e. [APTES] $=7.5 \mathrm{wt} . \%$ versus $0.25 \mathrm{wt} . \%$ for macro-scale double emulsions, see Fig. 6 (a)). Interestingly, for a given APTES concentration, the EE value depends on the SOR parameter. As a result, it is observed that the EE value and the size of the double nano-droplets are inversely correlated. This result is very important, even if the order of magnitude of the encapsulation efficiency is slightly below the ones of microscale double emulsions (discussed above Fig. 6), it remains absolutely unprecedented regarding nanoscale double droplets.
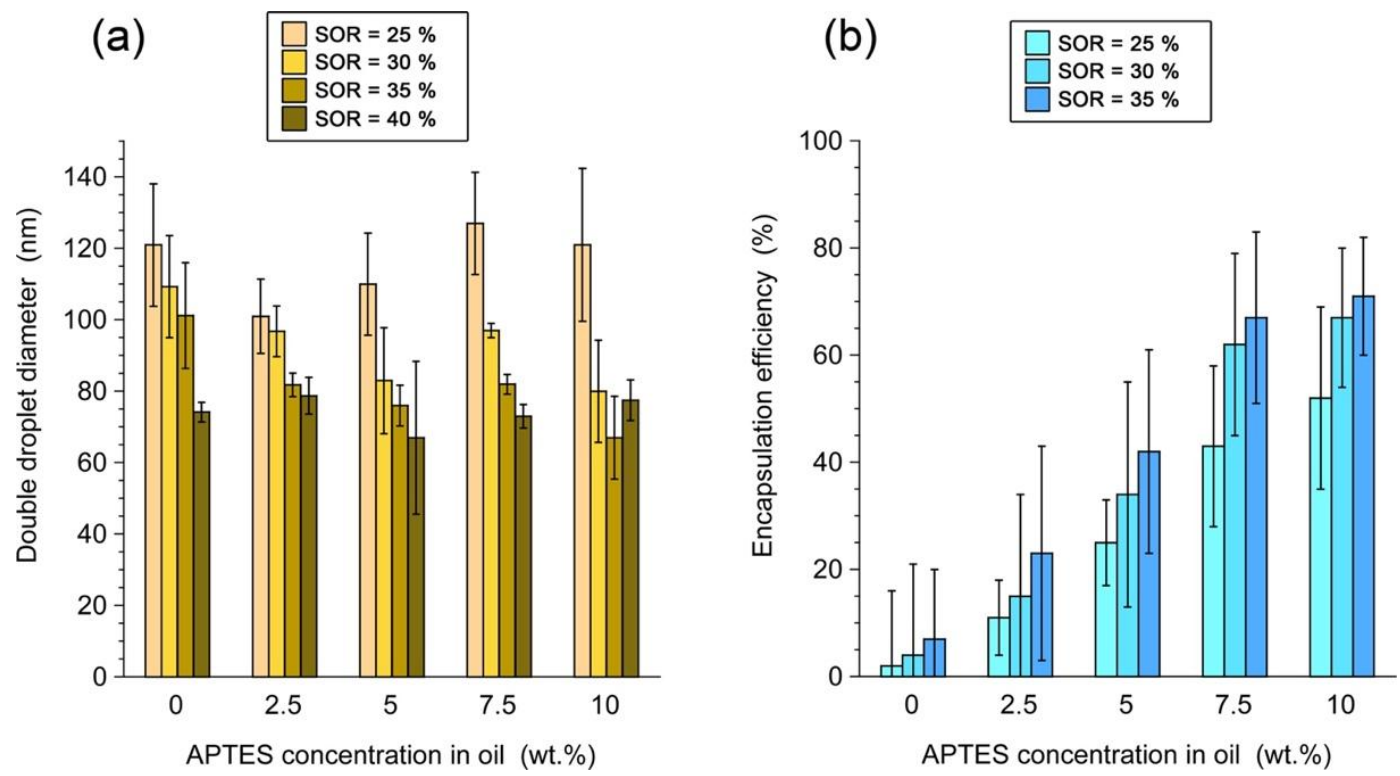
Figure 8: Diameter (a) and encapsulation efficiency (b) of double nano-emulsions for different concentrations of APTES and SOR. The nano-emulsions were obtained by spontaneous emulsification at room temperature. The encapsulation efficiencies were calculated from the concentration of methylene blue measured after application of fresh samples on size exclusion chromatography columns.

In order to confirm the double structure of the nano-emulsions, and further rationalize the data in Fig. 8, transmission election microscopy was performed on representative samples (Fig. 9).

(a)

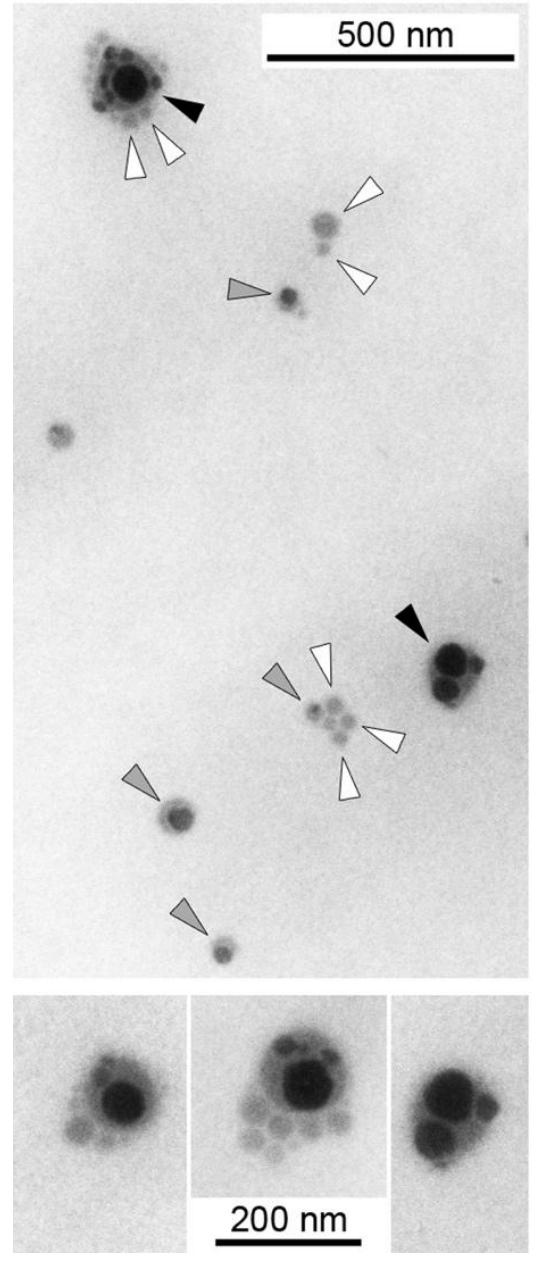

(b)

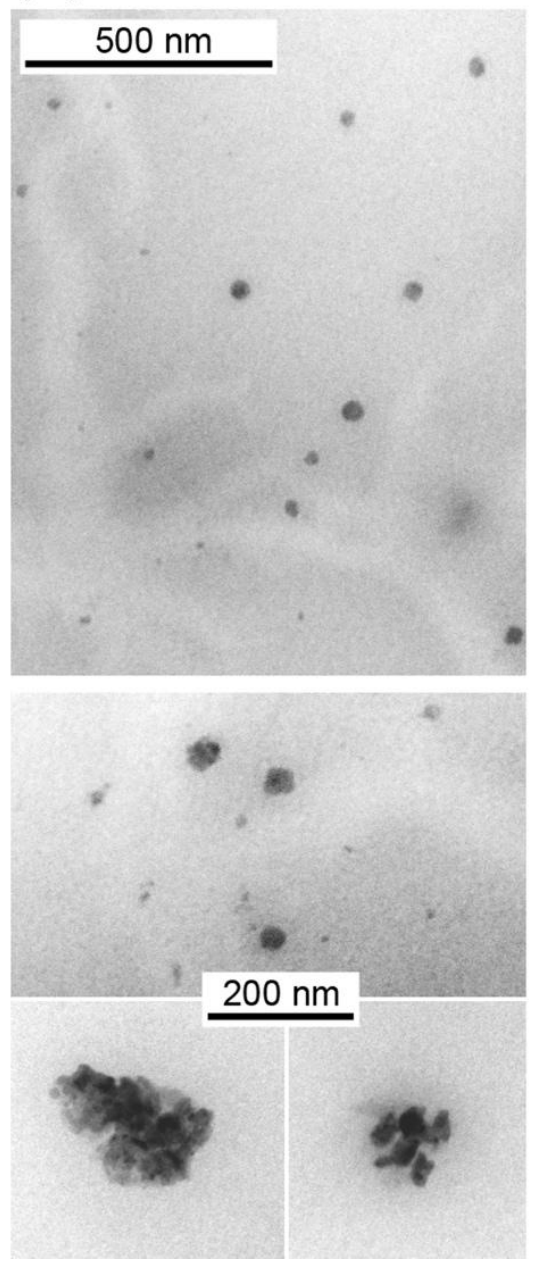

(c)

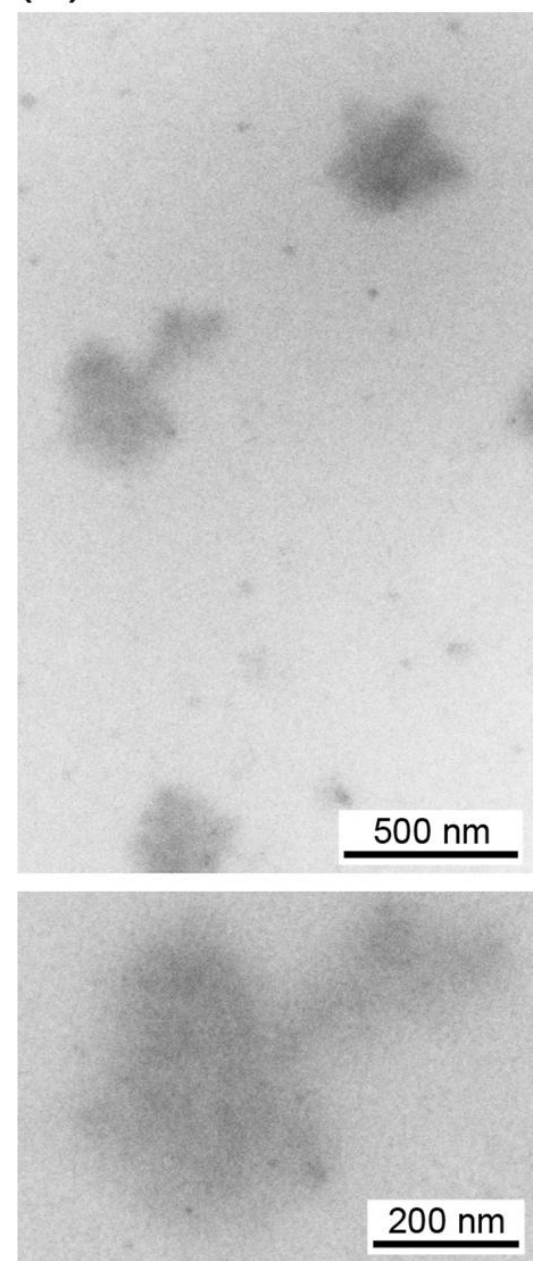

Figure 9: Transmission electron microscopy of double nano-emulsions for APTES concentrations of (a) 10 wt.\%, (b) 2.5 wt. $\%$, and (c) $0 \%$. Oil droplets can contain several (black arrowheads), one (gray arrowheads), or zero (white arrowheads) silica shell containing water droplets. 
The sizes of the double droplets correspond well to those in Fig. 8 (a), being centered at about $90 \mathrm{~nm}$ for $\mathrm{SOR}=30 \%$. The silica shells allow the droplets to conserve their shape upon drying under extensive vacuum on the carbon grid (Fig. 9 (a) and (b)). In contrast, the drying process in the absence of APTES destroys the nanodroplets and results in oil puddles (Fig. 9 (c)), as previously reported. ${ }^{54}$ For $[$ APTES] $=10 \mathrm{wt} . \%$ (Fig. 9 (a)), the $\sim 45 \mathrm{~nm}$ thickness of the silica shell (given in Fig. 4) appears strong enough to preserve the nano-droplet structure upon drying. The double nano-structure is clearly visible in this case, with the inner silica substructure in dark gray and the surrounding oil in light gray. Depending on their size, the double nano-droplets can include either several (black arrowheads), or only one (gray arrowheads) or no (white arrowheads) inner water droplets. The TEM pictures also reveal that the double droplets may be deformed by the primary droplets, showing their wettability by the oil, i.e. related to their good affinity to oil since they were not expelled out of the oily phase. Once bulk phase was removed, the capillary phenomenon should give the droplet final shapes and strongly impacts on the preservation of the droplet structure and shape. This is precisely what we observe, by comparing Fig. 9 (a) for which the structure is preserved thanks to the silica shells inside, and Fig. 9 (c) for which the oil spreads into the carbon support. Likewise, when several inner droplets are confined into one oil drop, they seem also deformed, forming a thin planar film at their contact region. These inter-droplets interactions in the bigger droplets, can probably lead to their small degradation compared to smaller one, explaining the difference in EE observed in function of their size (Fig. 8). The relatively dark aspect of the inner silica-stabilized droplets is likely attributed to the important thickness of the shell, as disclosed in Fig. 4. Very comparable visual dark aspects were previously reported according to similar sonochemistry of APTES at the 
water / oil interface of (this time) direct O/W nano-emulsions. ${ }^{55}$ This imagery seems related to the process, and to the thickness of the silica shell formed. This large silica shell (in Fig. 9 (a) around $45 \mathrm{~nm}$ ) can also explain why the encapsulation efficiency is not very high compared to the one expected in observing the double droplets by TEM: the water cavities are actually quite small. A remark can be done regarding the oil droplets containing or not internal particles, in fact, Fig. 9 (a) allows to show that only bigger droplets are able to have a double structure while smaller ones cannot. In fact, the spontaneous nano-emulsification gives rise to a log-normal size distribution, and logically, the inner silica shells can only be distributed in the second droplets big enough to entrap them.

On the other hand, nano-droplets with $[$ APTES] $=2.5$ wt.\% (Fig. 9 (b)) appear burst when water has escaped (during the sample drying), in line with their low EE values (Fig. 8 (b)) around $15 \%$ as compared to $70 \%$ for nano-droplets obtained with [APTES] $=10 \mathrm{wt} . \%$ (Fig. 9 (a)). The low EE value suggests that most of the inner water has escaped during the second nanoemulsification process. As a result, one can observe oil droplets containing remaining fragments of the shells. Noticeably, a good match is observed between the sizes measured by DLS (Fig. 4) and the TEM diameters of $130 \mathrm{~nm}$ for $[\mathrm{APTES}]=10 \%$, and $80 \mathrm{~nm}$ for $[\mathrm{APTES}]=2.5 \%$ (even if the droplets are destroyed, the scale appears coherent).

In addition to their encapsulation properties, an important feature of these new multifunctional nano-carriers is their ability to release and deliver an encapsulated material. To investigate this ability, we monitor the release of $\mathrm{MB}$ from double nano-emulsions incubated at $37^{\circ} \mathrm{C}$. The curves in Fig 10 represent the MB leakage into the external water phase $\left(\mathrm{W}_{2}\right)$ up to $6 \mathrm{~h}$ after formulation. As all the samples have the same initial amount of MB (initially in $\mathrm{w}_{1}$ ), but different EE values (Fig. 8 (b)), the initial offset corresponds to the MB that has already escaped during 
the second nano-emulsification. The release profiles are successfully fitted with a classical Korsmeyer-Peppas equation for spherical drug carriers, ${ }^{56} M_{t} / M_{0}=K_{m} t^{n}$, where $M_{t} / M_{0}$ is the fraction of drug release at time $t$, and $K_{m}$ a kinetic constant. The release exponent $n$ allows to identify the release regime.

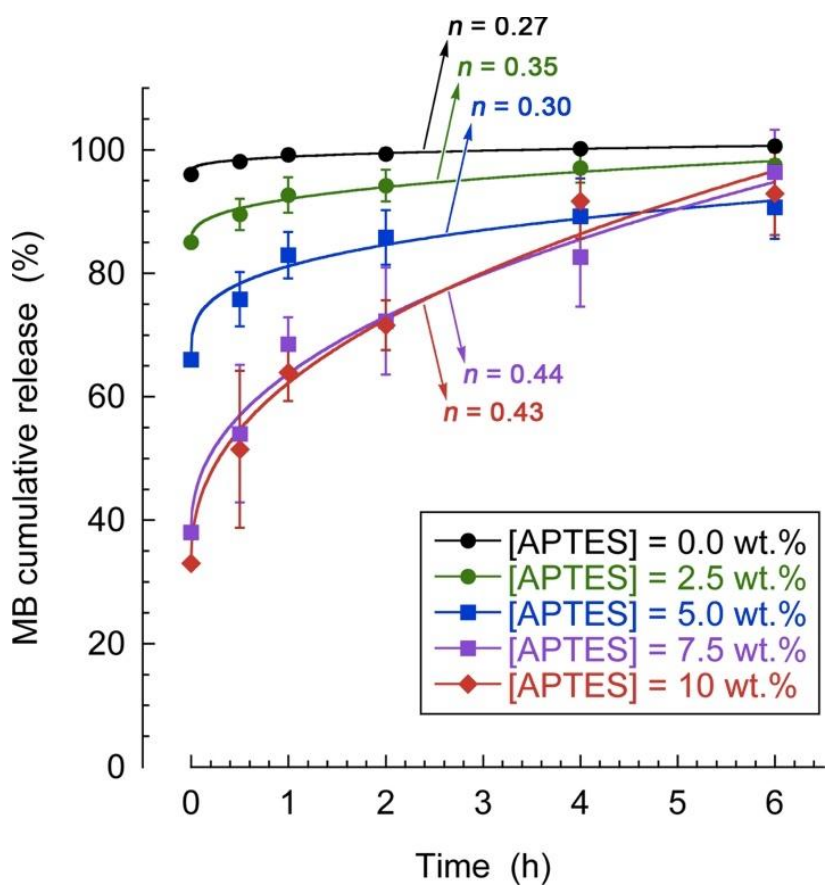

Figure 10: Release of methylene blue encapsulated in double nano-emulsions ( $\mathrm{SOR}=30 \%$ ). The Y-intercept value corresponds to the EE value after the second nano-emulsification. Experimental data are fitted using a KorsmeyerPeppas equation giving the values of the release exponent $n$, indicated in the figure.

For all samples, the encapsulated dye is effectively released (up to 100\%), indicating that the double nano-emulsions are affective nano-carriers able to deliver the encapsulated materials. All values of the release exponent $n$ (indicated in Fig. 10) remain below the threshold of 0.5 , indicating a Fickian diffusion. However, a difference should be made between the samples with $n \approx 0.44([\mathrm{APTES}]=7.5$ and $10 \mathrm{wt} . \%)$ indicative of a monodisperse Fickian drug release and 
the samples with $n \leq 0.35$ ([APTES] $=0,2.5$ and 5 wt.\%) indicative of a Fickian diffusion of polydisperse sample. ${ }^{56-58}$

This difference likely arises from the fact that the double emulsions are more fragile for the lower APTES concentrations, as evidenced by the disruption and fusion of their inner droplets observed by TEM images (Fig. 9). In contrast, the monodisperse Fickian drug release observed at higher APTES concentrations is in full line with the homogeneous population of internal droplets observed by TEM (Fig. 9).

Proving the presence of water in the inner droplets of micrometric and nanometric double emulsions $\mathrm{w}_{1} / \mathrm{O} / \mathrm{W}_{2}$

To achieve the characterization of double structure, beyond indirect ways like EE measurements or TEM observations, and to dispel doubts on the presence of water inside the double micro or nano- droplets, in this last paragraph we will use a water sensor to prove that inside the double droplets we still have liquid water. The water sensor is a fluorescent dye, sulforhodamine 101 (SR101), which presents a fluorescent signal only when it is solubilized in water. The general idea is to dissolve SR101 in the internal water $\left(\mathrm{w}_{1}\right.$, at $\left.100 \mu \mathrm{M}\right)$ right from the initial formulation stage, and to show that its fluorescent signal is still present within the double micro- or nanodroplets after their separation from the continuous phase. The whole experiment, along with spectrometric results are summarized in Fig. 11, showing the fluorescent spectra at every stages of the experiment. In addition, the control without silica was also reported in parallel to compare the spectra with the double emulsion with APTES $10 \mathrm{wt} . \%$. First, the fluorescent signal of 
reverse nano-emulsions is presented in Fig. 11 (a), showing a very important fluorescent signal with peaks at 606 and $607 \mathrm{~nm}$.

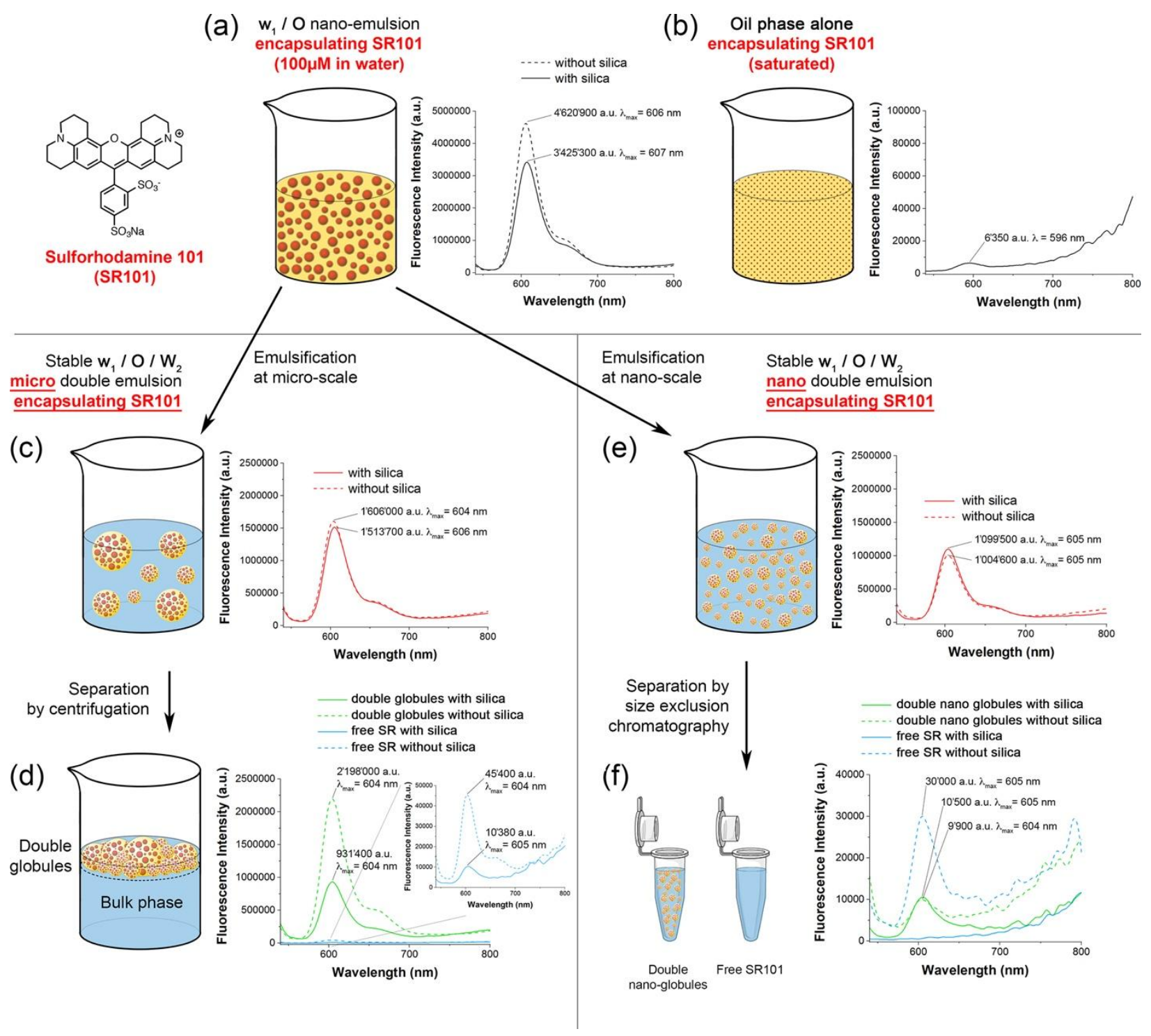

Figure 11: Schematic representation of the protocol followed to prove that liquid water is still present inside the micro- and nano- double droplets, along with the corresponding fluorescent spectra. Sulforhodamine (SR101) is encapsulated in the $\mathrm{w}_{1} / \mathrm{O}$ nano-emulsions (a) with and without silica (APTES $10 \%$ in oil), (b) dispersed at saturation in oil as a control, and then encapsulated in micro double droplets before (c) and after (d) separation by centrifugation, or encapsulated in nano double droplets before (e) and after (f) separation by size exclusion chromatography. 
At exactly the same concentration, the two signals with and without APTES show a significantly different fluorescence (around $4.6 \times 10^{6}$ a.u. and $3.4 \times 10^{6}$ a.u., respectively), likely due to the change of composition that generally affects the environment of the dyes and can modify their fluorescent properties. On the other hand, when dispersed in oil at saturation (Fig. 11 (b)), SR101 displays a much lower fluorescent signal of few orders of magnitudes (around $6 \times 10^{3}$ ) not comparable with the former, and -important for the discussion- slightly color-shifted around $10 \mathrm{~nm}$ at $596 \mathrm{~nm}$. This huge difference between water and oil signals, compared to the fraction containing double droplets and free dyes, will indicate whether SR101 is still dissolved in water or not, after separation of the droplets from the bulk.

The formulation of micro-scale double emulsions is shown in Fig. 11 (c) and (d), before and after droplets separations, respectively. Before separation (Fig. 11 (c)), both signals are comparable (around $1.5 \times 10^{6}$ a.u.), in the line with the curves of primary emulsion (Fig. 11 (a)) diluted by the formulation process (with the difference between with and without silica still visible). Then, the droplet separation shows a much more important signal in the double droplets compared to the bulk phase, higher than $1.5 \times 10^{6}$ a.u. and lower than $6 \times 10^{3}$ a.u., respectively. The wavelength of the peak $\left(\lambda_{\max }=604\right.$ and $605 \mathrm{~nm}$ ), as well, corresponds to the signal of SR101 solubilized in water and not transferred to oil (see Fig. 11 (a) and (b)). This first result proves that SR101 is still and importantly solubilized in water within the microscale double droplets, thus in the internal water. Interestingly the signal with and without silica exhibit different fluorescence intensity, higher in both cases corresponding to the samples without silica. This is due to the dye leakage from the double droplets without silica (EE is around 22\%) when there is no leakage with silica (EE around 100\%), see Fig. 6 (a). This gives rise to the dequenching of SR101 inside the droplets (due to the ACQ effect as it is explained in the Experimental section), along with an 
increase of the SR101 concentration in the bulk phase, thus increasing the fluorescent signal in both cases. In addition, a close study of the different fluorescent intensities discloses a decrease of the dye concentration inside the internal droplets, likely due to the dye escape. For example, with "micro-droplets with silica", the dilution factor inherent to the double droplet's formulation

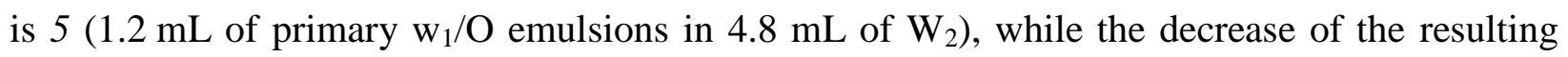
fluorescent signal is only around $2.2\left(1.5 \times 10^{6}\right.$ a.u. $/ 3.4 \times 10^{6}$ a.u. $)$. This increase goes in the sense of a dye dequenching due to a concentration decrease, i.e. due to the dye release from the inner droplets (as observed with methylene blue).

On the other hand, in the case of nano-double emulsions, SR101 signal in double nano-emulsions arises slightly more diluted around $1.5 \times 10^{6}$ a.u. (Fig. 11 (e)), due to the formulation method, however this dilution is even further increased after the size exclusion chromatography separation (Fig. 11 (f)). The fluorescent signal in double nano-droplets with and without silica still appears measurable and significative (around $1.0 \times 10^{4}$ a.u.), and importantly, the peaks maximums appear at 604 and $605 \mathrm{~nm}$ (and not at $596 \mathrm{~nm}$ when the dye is dispersed in oil in Fig. 11 (b)). These two points confirm that SR101 is still solubilized in water, inside the double nano-droplets. Then, the signal of the free dyes appears important in the fraction without silica, and inexistent with silica, in line with the former EE results and conclusions established in the previous sections.

\section{Conclusion}

In this study, we formulate stable double emulsions from the micrometric to the nanometric scale, with respective properties. This is achieved by simply reinforcing the water / oil interface of the primary internal water droplet with a silica membrane, resulting in efficient encapsulation 
of the hydrophilic materials in the internal water. We show that the modulation of the thickness of the silica membrane (by varying the formulation parameters) can modulate the encapsulation efficiency (up to $100 \%$ encapsulation), size and release properties. Such a system paves the way for a large number of applications from pharmaceutics with delivery of hydrophilic active principles, to agri-food and cosmetics applications with the long-term storage and protection of fragile, instable or volatile compounds. Moreover, the double emulsion morphology transposed to the nanoscale has never been reported and is a novel solution for the i.v. administration and targeted co-administration of hydrophilic and lipophilic agents.

\section{Acknowledgments}

The authors acknowledges the Higher Education Commission of Pakistan for providing financial support of the $\mathrm{PhD}$ scholar S.A.

\section{References}

(1) Lamba, H.; Sathish, K.; Sabikhi, L. Double Emulsions: Emerging Delivery System for Plant Bioactives. Food Bioprocess Technol. 2015, 8 (4), 709-728.

(2) Hearn, T. L.; Olsen, M.; Hunter, R. L. Multiple Emulsions Oral Vaccine Vehicles for Inducing Immunity or Tolerance. Ann. N. Y. Acad. Sci. 1996, 778 (1), 388-389.

(3) Okochi, H.; Nakano, M. Preparation and Evaluation of w/o/w Type Emulsions Containing Vancomycin. Adv. Drug Deliv. Rev. 2000, 45 (1), 5-26.

(4) Muschiolik, G.; Dickinson, E. Double Emulsions Relevant to Food Systems: Preparation, Stability, and Applications. Compr. Rev. Food Sci. Food Saf. 2017, 16 (3), 532-555.

(5) Assadpour, E.; Maghsoudlou, Y.; Jafari, S.-M.; Ghorbani, M.; Aalami, M. Evaluation of Folic Acid Nano-Encapsulation by Double Emulsions. Food Bioprocess Technol. 2016, 9 (12), 20242032.

(6) Esfanjani, A. F.; Jafari, S. M.; Assadpoor, E.; Mohammadi, A. Nano-Encapsulation of Saffron Extract through Double-Layered Multiple Emulsions of Pectin and Whey Protein Concentrate. $J$. 
Food Eng. 2015, 165, 149-155.

(7) Esfanjani, A. F.; Jafari, S. M.; Assadpour, E. Preparation of a Multiple Emulsion Based on PectinWhey Protein Complex for Encapsulation of Saffron Extract Nanodroplets. Food Chem. 2017, 221, 1962-1969.

(8) Mohammadi, A.; Jafari, S. M.; Assadpour, E.; Esfanjani, A. F. Nano-Encapsulation of Olive Leaf Phenolic Compounds through WPC-Pectin Complexes and Evaluating Their Release Rate. Int. J. Biol. Macromol. 2016, 82, 816-822.

(9) Matsumoto, S. Development of W/O/W-Type Dispersion during Phase Inversion of Concentrated W/O Emulsions. J. Colloid Interface Sci. 1983, 94 (2), 362-368.

(10) Pradhan, M.; Rousseau, D. A One-Step Process for Oil-in-Water-in-Oil Double Emulsion Formation Using a Single Surfactant. J. Colloid Interface Sci. 2012, 386 (1), 398-404.

(11) O’ Dwyer, S. P.; O’ Beirne, D.; Ní Eidhin, D.; Hennessy, A. A.; O’ Kennedy, B. T. Formation, Rheology and Susceptibility to Lipid Oxidation of Multiple Emulsions (O/W/O) in Table Spreads Containing Omega-3 Rich Oils. LWT - Food Sci. Technol. 2013, 51 (2), 484-491.

(12) Ding, S.; Anton, N.; Akram, S.; Er-Rafik, M.; Anton, H.; Klymchenko, A.; Yu, W.; Vandamme, T. F. T. F.; Serra, C. A. C. A. A New Method for the Formulation of Double Nanoemulsions. Soft Matter 2017, 13 (8), 1660-1669.

(13) Zhang, M.; Corona, P. T.; Ruocco, N.; Alvarez, D.; Malo de Molina, P.; Mitragotri, S.; Helgeson, M. E. Controlling Complex Nanoemulsion Morphology Using Asymmetric Cosurfactants for the Preparation of Polymer Nanocapsules. Langmuir 2018, 34 (3), 978-990.

(14) Malo de Molina, P.; Zhang, M.; Bayles, A. V.; Helgeson, M. E. Oil-in-Water-in-Oil Multinanoemulsions for Templating Complex Nanoparticles. Nano Lett. 2016, 16 (12), 7325 7332.

(15) Zhang, M.; Nowak, M.; Malo de Molina, P.; Abramovitch, M.; Santizo, K.; Mitragotri, S.; Helgeson, M. E. Synthesis of Oil-Laden Poly(Ethylene Glycol) Diacrylate Hydrogel Nanocapsules from Double Nanoemulsions. Langmuir 2017, 33 (24), 6116-6126.

(16) Florence, A. T.; Whitehill, D. The Formulation and Stability of Multiple Emulsions. Int. J. Pharm. 1982, 11 (4), 277-308.

(17) Hanson, J. A.; Chang, C. B.; Graves, S. M.; Li, Z.; Mason, T. G.; Deming, T. J. Nanoscale Double Emulsions Stabilized by Single-Component Block Copolypeptides. Nature 2008, 455 (7209), 8588.

(18) Mehrnia, M.-A.; Jafari, S.-M.; Makhmal-Zadeh, B. S.; Maghsoudlou, Y. Rheological and Release Properties of Double Nano-Emulsions Containing Crocin Prepared with Angum Gum, Arabic Gum and Whey Protein. Food Hydrocoll. 2017, 66, 259-267.

(19) Zhao, Y.; Zhang, J.; Li, W.; Zhang, C.; Han, B. Synthesis of Uniform Hollow Silica Spheres with Ordered Mesoporous Shells in a CO2 Induced Nanoemulsion. Chem. Commun. 2009, No. 17, 2365-2367.

(20) Wu, S.-H.; Hung, Y.; Mou, C.-Y. Compartmentalized Hollow Silica Nanospheres Templated from Nanoemulsions. Chem. Mater. 2013, 25 (3), 352-364.

(21) Spernath, L.; Magdassi, S. Formation of Silica Nanocapsules from Nanoemulsions Obtained by the Phase Inversion Temperature Method. Micro Nano Lett. 2010, 5 (1), 28-36. 
(22) Attia, M. F.; Anton, N.; Bouchaala, R.; Didier, P.; Arntz, Y.; Messaddeq, N.; Klymchenko, A. S.; Mély, Y.; Vandamme, T. F. Functionalization of Nano-Emulsions with an Amino-Silica Shell at the Oil-Water Interface. RSC Adv. 2015, 5, 74353-74361.

(23) Hu, Y.; Zou, S.; Chen, W.; Tong, Z.; Wang, C. Mineralization and Drug Release of Hydroxyapatite/Poly(l-Lactic Acid) Nanocomposite Scaffolds Prepared by Pickering Emulsion Templating. Colloids Surfaces B Biointerfaces 2014, 122, 559-565.

(24) Nebogina, N. A.; Prozorova, I. V.; Yudina, N. V. Effect of Mineralization of Aqueous Phase and Watering on the Composition of the Interphase Layer of Water-Oil Emulsions. Chem.Sust. Dev. 2009, 17, 207-212.

(25) Fornasieri, G.; Badaire, S.; Backov, R. V.; Poulin, P.; Zakri, C.; Mondain-Monval, O. Mineralization of Water-in-Oil Emulsions Droplets. Mater. Res. Soc. Symp. Proc. 2005, 847 (1$6)$.

(26) Kresge, C. T.; Loenowicz, M. E.; Roth, W. J.; Vartulli, J. C.; Beck, J. S. Ordered Mesoporous Molecular Sieves Synthesized by a Liquid-Crystal Template Mechanism. Nature 1992, 359, 710712.

(27) Cacace, D. N.; Rowland, A. T.; Stapleton, J. J.; Dewey, D. C.; Keating, C. D. Aqueous Emulsion Droplets Stabilized by Lipid Vesicles as Microcompartments for Biomimetic Mineralization. Langmuir 2015, 31 (41), 11329-11338.

(28) Jutz, G.; Böker, A. Bio-Inorganic Microcapsules from Templating Protein- and BionanoparticleStabilized Pickering Emulsions. J. Mater. Chem. 2010, 20 (21), 4299-4304.

(29) Liberman, A.; Mendez, N.; Trogler, W. C.; Kumme, A. C. Synthesis and Surface Functionalization of Silica Nanoparticles for Nanomedicine. Surf. Sci. Rep. 2014, 69 (2-3), 132 158.

(30) Jaganathan, H.; Godin, B. Biocompatibility Assessment of Si-Based Nano- and Micro-Particles. Adv. Drug. Deliv. Rev. 2012, 64 (15), 1800-1819.

(31) Foglia, S.; Ledda, M.; Fioretti, D.; Iucci, G.; Papi, M.; Capellini, G.; Lolli, M. G.; Grimaldi, S.; Rinaldi, M.; Lisi, A. In Vitro Biocompatibility Study of Sub-5 Nm Silica-Coated Magnetic Iron Oxide Fluorescent Nanoparticles for Potential Biomedical Application. Sci. Rep. 2017, 7, 46513.

(32) Anton, N.; Vandamme, T. F. T. F. The Universality of Low-Energy Nano-Emulsification. Int. J. Pharm. 2009, 377 (1), 142-147.

(33) Anton, N.; Benoit, J.-P.; Saulnier, P. Design and Production of Nanoparticles Formulated from Nano-Emulsion Templates-A Review. J. Control. Release 2008, 128 (3).

(34) Anton, N.; Vandamme, T. F. Nano-Emulsions and Micro-Emulsions: Clarifications of the Critical Differences. Pharm. Res. 2011, 28 (5), 978-985.

(35) Ocotlán-Flores, J.; Saniger, J. M. Catalyst-Free SiO2 Sonogels. J. Sol-Gel Sci. Technol. 2006, 39 (3), 235-240.

(36) Kentish, S.; Wooster, T. J.; Ashokkumar, M.; Balachandran, S.; Mawson, R.; Simons, L. The Use of Ultrasonics for Nanoemulsion Preparation. Innov. Food Sci. Emerg. Technol. 2008, 9 (2), 170175.

(37) Mahdi Jafari, S.; He, Y.; Bhandari, B. Nano-Emulsion Production by Sonication and Microfluidization-A Comparison. Int. J. Food Prop. 2006, 9 (3), 475-485. 
(38) Anton, N.; Benoit, J.-P.; Saulnier, P. Design and Production of Nanoparticles Formulated from Nano-Emulsion Templates-A Review. J. Control. Release 2008, 128 (3), 185-199.

(39) Delmas, T.; Piraux, H.; Couffin, A.-C.; Texier, I.; Vinet, F.; Poulin, P.; Cates, M. E.; Bibette, J. How To Prepare and Stabilize Very Small Nanoemulsions. Langmuir 2011, 27 (5), 1683-1692.

(40) Tang, S. Y.; Shridharan, P.; Sivakumar, M. Impact of Process Parameters in the Generation of Novel Aspirin Nanoemulsions - Comparative Studies between Ultrasound Cavitation and Microfluidizer. Ultrason. Sonochem. 2013, 20 (1), 485-497.

(41) Gaikwad, S. G.; Pandit, A. B. Ultrasound Emulsification: Effect of Ultrasonic and Physicochemical Properties on Dispersed Phase Volume and Droplet Size. Ultrason. Sonochem. 2008, 15 (4), 554-563.

(42) Manchun, S.; Dass, C. R.; Sriamornsak, P. Designing Nanoemulsion Templates for Fabrication of Dextrin Nanoparticles via Emulsion Cross-Linking Technique. Carbohydr. Polym. 2014, 101, $650-655$.

(43) Abismaïl, B.; Canselier, J. P.; Wilhelm, A. M.; Delmas, H.; Gourdon, C. Emulsification by Ultrasound: Drop Size Distribution and Stability. Ultrason Sonochem. 1999, 6 (1-2), 75-83.

(44) Marquez, A. L.; Wagner, J. R. Rheology of Double (W/O/W) Emulsions Prepared with Soybean Milk and Fortified with Calcium. J. Texture Stud. 2010, 41 (5), 651-671.

(45) Gupta, A.; Eral, H. B.; Hattona, T. A.; Doyle, P. S. Controlling and Predicting Droplet Size of Nanoemulsions: Scaling Relations with Experimental Validation. Soft Matter 2015, 12, 14511458.

(46) Gupta, A.; Eral, H. B.; Hatton, T. A.; Doyle, P. S. Nanoemulsions: Formation, Properties and Applications. Soft Matter 2016, 12 (11), 2826-2841.

(47) Márquez, A. L.; Medrano, A.; Panizzolo, L. A.; Wagner, J. R. Effect of Calcium Salts and Surfactant Concentration on the Stability of Water-in-Oil (w/o) Emulsions Prepared with Polyglycerol Polyricinoleate. J. Colloid Interface Sci. 2010, 341 (1), 101-108.

(48) Luo, H.; Macapagal, N.; Newell, K.; Man, A.; Parupudi, A.; Li, Y.; Li, Y. Effects of Salt-Induced Reversible Self-Association on the Elution Behavior of a Monoclonal Antibody in Cation Exchange Chromatography. J Chromatogr A 2014, 1362, 186-193.

(49) Lee, L.; Hancocks, R.; Noble, I.; Norton, I. T. Production of Water-in-Oil Nanoemulsions Using High Pressure Homogenisation: A Study on Droplet Break-Up. J. Food Eng. 2014, 131, 33-37.

(50) Wibowo, D.; Zhao, C. X.; Middelberg, A. P. Emulsion-Templated Silica Nanocapsules Formed Using Bio-Inspired Silicification. Chem Commun 2014, 50 (77), 11325-11328.

(51) Jakhmola, A.; Vecchione, R.; Guarnieri, D.; Belli, V.; Calabria, D.; Netti, P. A. Bioinspired Oil Core/Silica Shell Nanocarriers with Tunable and Multimodal Functionalities. Adv. Healthc. Mater. 2015, 4 (17), 2688-2698.

(52) Saberi, A. H.; Fang, Y.; McClements, D. J. Fabrication of Vitamin E-Enriched Nanoemulsions: Factors Affecting Particle Size Using Spontaneous Emulsification. J. Colloid Interface Sci. 2013, $391,95-102$.

(53) Guttoff, M.; Saberi, A. H.; McClements, D. J. Formation of Vitamin D Nanoemulsion-Based Delivery Systems by Spontaneous Emulsification: Factors Affecting Particle Size and Stability. Food Chem. 2015, 171, 117-122. 
(54) Attia, M. F.; Anton, N.; Akasov, R.; Chiper, M.; Markvicheva, E.; Vandamme, T. F. Biodistribution and Toxicity of X-Ray Iodinated Contrast Agent in Nano-Emulsions in Function of Their Size. Pharm. Res. 2016, 33 (3), 603-614.

(55) Attia, M. F.; Anton, N.; Bouchaala, R.; Didier, P.; Arntz, Y.; Messaddeq, N.; Klymchenko, A. S.; Mély, Y.; Vandamme, T. F. Functionalization of Nano-Emulsions with an Amino-Silica Shell at the Oil-Water Interface. RSC Adv. 2015, 5 (91).

(56) Ritger, P. L.; Peppas, N. A. A Simple Equation for Description of Solute Release I. Fickian and Non-Fickian Release from Non-Swellable Devices in the Form of Slabs, Spheres, Cylinders or Discs. J. Control. Release 1987, 5, 23-36.

(57) Gupta, A.; Badruddoza, Z.; Doyle, P. S. A General Route for Nanoemulsion Synthesis Using Low Energy Methods at Constant Temperature. Langmuir 2017, 33 (29), 7118-7123.

(58) Badruddoza, A. Z.; Gupta, A.; Myerson, A. S.; Trout, B. L.; Doyle, P. S. Low Energy Nanoemulsions as Templates for the Formulation of Hydrophobic Drugs. Adv. Ther. 2018, 1700020 .

\section{Graphical Table of Content}

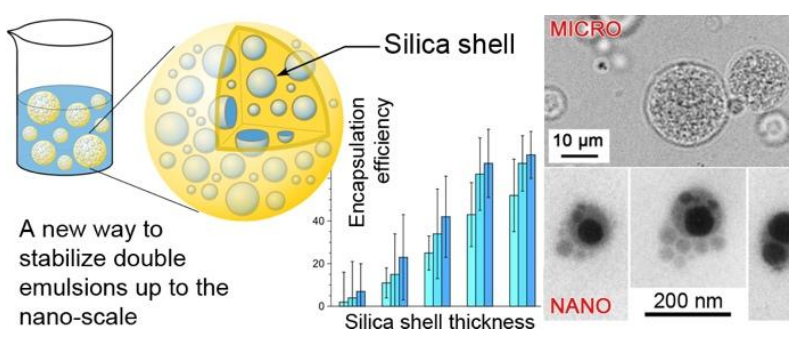

